\title{
CARNOSINE AND HOMOCARNOSINE
}

\author{
DEGRATION MECHANISMS BY THE
}

\section{HUMAN CARNOSINASE ENZYME CN1:}

\section{INSIGHTS FROM MULTISCALE}

\section{SIMULATIONS}

Matic Pavlin ${ }^{1,2}$, Giulia Rossetti ${ }^{2,3,4 *}$, Marco De Vivo ${ }^{2,5}$, Paolo Carloni $^{1,2}$

${ }^{1}$ Laboratory for Computational Biophysics, German Research School for Simulation Sciences (GRS), Forschungszentrum Jülich - RWTH Aachen, 52425 Jülich, Germany

${ }^{2}$ Computational Biomedicine section (INM-9), Institute for Neuroscience and Medicine (INM), and (IAS-5), Institute of Advanced Simulation (IAS), Forschungszentrum Jülich, 52425 Jülich, Germany

${ }^{3}$ Jülich Supercomputing Center (JSC), Forschungszentrum Jülich, 52425 Jülich, Germany

${ }^{4}$ Department of Oncology, Hematology and Stem Cell Transplantation, University Hospital Aachen, RWTH Aachen University, Pauwelsstraße 30, 52074 Aachen, Germany

${ }^{5}$ Laboratory of Molecular Modeling and Drug Discovery, Istituto Italiano di Tecnologia, Via Morego 30, 16163 Genoa, Italy

*E-mail: g.rossetti@fz-juelich.de 


\section{SUPPORTING INFORMATION}

\section{Hess analysis for convergence.}

The cosine content of the first principal components (PCs) is an indicator for sampling in molecular dynamics (MD) simulations. ${ }^{1}$ A cosine content close to 1 indicates bad sampling, while a value close to 0 suggests that the simulated time was long enough to sufficiently sample the system. The Hess analysis of both Hcar and Lcar MD simulations suggests that the latter is the case in our simulations (see Tab. S5).

\section{Chemical shifts calculations.}

We followed the procedure of ref. ${ }^{2}$ First, structures of NMR experimental standards (tetramethylsilane (TMS) for ${ }^{1} \mathrm{H}$ and ${ }^{13} \mathrm{C}$ and sodium [2,2,3,3-2H4]-3trimethylsilylpropanoate for ${ }^{1} \mathrm{H}$ in Lcar and sodium 2,2-dimethyl-2-silanpentane-5-sulfonate (DSS) for ${ }^{1} \mathrm{H}$ in Hcar) were optimized on the B3LYP/6-31G(d,p) level of theory. Chemical shifts of the standards' structures along with representative structures 1-4A, 1-4B, 1-4C and 1-4D (for definition see Chart 1 in the Main Text) were calculated at the B3LYP/6$311+G(2 d, p)$ level of theory. Finally, the calculated chemical shift of each hydrogen and carbon atom in each representative structure was subtracted from the corresponding calculated chemical shift of standards. The Gaussian09 software package was used for these calculations. ${ }^{3}$

\section{Lcar and Hcar in water.}

The four highest populated clusters for each tautomer of Lcar (1A-4A, 1B-4B hereafter) cover $\sim 70 \%$ of the total conformational space. $\mathbf{1 A}$ and $\mathbf{3 A}$ (covering in total the $54 \%$ of the simulated time of $\mathbf{A}$ ) assume the 'extended' conformation, while $\mathbf{2 A}$ and $\mathbf{4 A}$ feature a 'semifolded $^{14}$ conformation. The populations of the 'extended' and 'semi-folded' conformations are comparable, as reported in ref. ${ }^{4}$ (Fig. S5).

$\mathbf{1 B}$ and $\mathbf{2 B}$ ( $81 \%$ of the simulated time of $\mathbf{B})$ are 'extended', while $\mathbf{3 B}$ and $\mathbf{4 B}$ have a 'semifolded' conformation (Fig. S5), showing a predominance of 'extended' conformations for this Lcar tautomer (Tab. 1) This is fully consistently with experiments ${ }^{5}$ and quantum chemical calculations. ${ }^{6}$

The four highest populated clusters of tautomer $\mathbf{C}$ (1C-4C) of Hcar cover $61 \%$ of the simulated time, while for tautomer D (1D-4D) this value is increased to $74 \%$. The highest populated cluster of tautomer $\mathbf{D}$ is, analogously to Lcar, in 'extended' conformation (see Fig. S5).

From a histogram of the RMSF as function of atom number, we suggest that among the two tautomers of Lcar A is more flexible than $\mathbf{B}$ in solution (Fig. S6).

\section{Docking of Lcar and Hcar to the hCN1 monomer.}

We first docked Lcar (B tautomer) and Hcar (D tautomer) onto refined apo monomeric hCN1 X-ray structure by using GOLD code. ${ }^{7}$ Here we observed the shift of the bridging water molecule for an average value of $1.6 \AA$ (see Tab. S7). The poses were then refined by performing AutoDock calculations ${ }^{8}$ with an improved force field for zinc proteins ${ }^{9}$ ).

hCN1 $\boldsymbol{\alpha}$. Docking calculations of Lcar to the hCN1 $\boldsymbol{\alpha}$ structure ( $\boldsymbol{\alpha}$ stands for tautomeric state of hCN1 where Glu173 is protonated at Oe2 carboxylic oxygen (Glh173 $\alpha$ ); see Fig. 2 in the Main Text) returned two different positions of Lcar. These differ in the orientation of $\beta-$ alanine part. In one structure it is folded, while in the other one it is extended. However, both Lcar's conformers form the same hydrogen bonds, namely histidine ring of Lcar with backbone oxygen of Glu174, peptide NH group with backbone oxygen of Thr424 and 
carboxylic group with peptide NH group of Thr424. Additionally, Lcar's carboxylic group forms a salt bridge with side chain of Arg350 and the peptide oxygen of Lcar forms a hydrogen bond with the bridging hydroxyl ion (see also Tab. S18). In the complex where Lcar is in extended conformation, the substrate's orientation seems more prone to the hydrolysis: Namely, when Lcar is in folded conformation, amino terminal group is orientated towards Glh173 (Glh173 stands for protonated Glu173), which plays an active role in the hydrolysis. This orientation of Lcar's amino group could hamper the course of the reaction. On the other hand, Lcar in extended conformation does not feature any steric hindrances for the hydrolysis to take place and moreover, it could be additionally stabilized with hydrogen bonds between its amino group and nearby residues (Asp202, Asn203 or Asp421) (see Fig. S8). Moreover, as reported above, the extended conformation is the most populated one in solution according to Maxwell-Boltzmann statistics. Therefore, we used structure of this complex for further calculations.

A similar interaction pattern to the above-discussed Lcar, is also observed Hcar docked into hCN1. The key difference between the two dipeptides is that Hcar forms an additional hydrogen bond between its amino group and side chain of Asn203. Due to this additional interaction, Hcar is present inside the hCN1 active site only in the extended form.

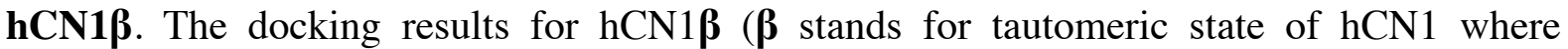
Glu173 is protonated at Oe1 carboxylic oxygen; see Fig. 2 in the Main Text) show slightly different binding poses and interaction network, with respect to the previous one. Namely, Lcar is found only in extended conformation stabilized by a hydrogen bond between its amino group and Asn203 side chain, which is not present in hCN1 $\alpha$. On the other hand, the hydrogen bond between backbone oxygen of Glu174 and histidine ring of Lcar is not present anymore. For Hcar, we got the same set of interactions as in hCN1 $\alpha$ with additional hydrogen bond between its amino group and backbone oxygen of Asp202 (see also Tab. S18).

Additional docking calculations. We performed also docking calculations with the other

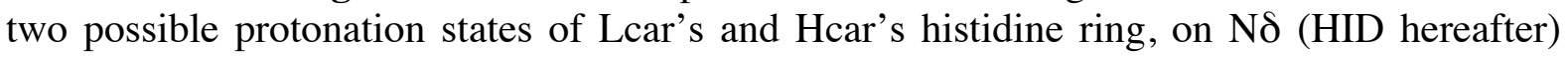
and on both, the $\mathrm{N} \delta$ and the $\mathrm{N} \varepsilon$ atoms (HIP hereafter).

In the case of Lcar, the three protonation states result in similar binding poses. The difference is only in a hydrogen bond formed between the ligand's imidazole ring and hCN1: in the case of HIE and HIP, imidazole ring hydrogen bonds to Glu174 backbone oxygen, while in HID imidazole ring forms hydrogen bond with the side chain of Glh173 (see also Fig. S9 and Tab. S18). The RMSD of non-hydrogen atoms of HID and HIP protonation states with respect to that of HIE are $2.1 \AA$ and $1.3 \AA$, respectively. The RMSD of the peptide bond in HID and HIP relative to HIE is $0.4 \AA$ and $0.6 \AA$.

Also in the case of Hcar, the three protonation states result in similar binding poses. However, both HID and HIP form in total one hydrogen bond less with hCN1 than HIE tautomer. Specifically, HID imidazole ring forms no hydrogen bonds with hCN1. HIP ligand's peptide's NH group forms no hydrogen bond with Thr424 backbone. The amino terminal group forms a hydrogen bond with Asp202 (see also Fig. S9 and Tab. S18). Therefore, both HID and HIP protonation states appeared less stabilized than HIE. RMSD of ${ }^{\text {a }}$ Please note that the latter interactions where not observed in the docking calculations, however some of them were present in the
subsequent QM/MM simulations. 
non-hydrogen atoms of HID and HIP protonation states with respect to HIE is $1.1 \AA$ and 1.4 $\AA$, respectively, while the RMSD of the peptide bond in HID and HIP relative to HIE is $0.3 \AA$ and $0.5 \AA$.

\section{QM/MM simulations of Lcar $\bullet h C N 1 \beta$ and Hcar $\bullet h C N 1 \beta$ Michaelis complexes.}

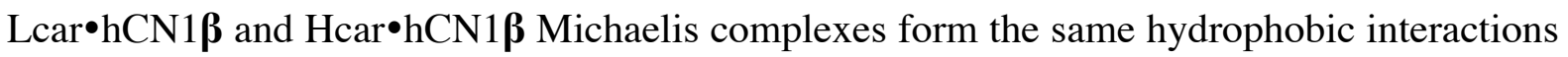
as in hCN1 $\alpha$, while few subtle differences for hydrogen bonds and salt bridges can be observed. Specifically, for Lcar, amino terminal group hydrogen bonds to backbone of Asp202 and the Lcar's amide hydrogen bond with Thr424 backbone oxygen are more persistent than the same ones observed in hCN1 $\alpha$ (see Tab. S23, Tab. 2 and Fig. S11). Further, the hydrogen bond between Lcar's carboxyl group and Thr450 backbone unit is direct and not water-mediated as in $\mathrm{hCN} 1 \boldsymbol{\alpha}$ (despite being slightly less persistent). Finally, the Lcar's amino terminal group salt bridge with Asp202, observed in hCN1 $\boldsymbol{\alpha}$, is now absent, while Lcar's salt bridge with Arg350 side chain is more persistent.

In Hcar $\bullet$ hCN1 $\boldsymbol{\beta}$, Hcar's carbonylic oxygen forms a hydrogen bond with Glh173 side chain. Hcar's amino terminal group forms hydrogen bonds with Asn203 and Ser423 side chains. These interactions are absent in hCN1 $\alpha$ (see Tab. S23 and Tab. 2). Hcar's amino terminal group forms also a water-mediated hydrogen bond with Asp202 backbone. In hCN1 $\alpha$, this Hbond is direct. The hydrogen bond between Hcar's amide and Thr424 backbone oxygen is less persistent than that in hCN1 $\boldsymbol{\alpha}$. Hcar's carboxyl group does not form an H-bond with Thr424 backbone unit as in hCN1 $\alpha$ (see Tab. S23 and Tab. 2). Hcar's amino terminal group forms a persistent salt bridge with Asp421, while in hCN1 $\boldsymbol{\alpha}$ there is water-mediated hydrogen bond between those two residues. The Hcar's salt bridge with Arg350 side chain is less persistent than in hCN1 $\alpha$ (see Tab. S23 and Tab. 2).

Hcar forms one more hydrogen bond with hCN1 than Lcar. Therefore the flexibility of the ligand in the binding site is larger for Lcar than that of Hcar (see Fig. S10).

As in hCN1 $\alpha$, the $\mathrm{Zn}^{2+}$ ions polyhedra are similar to those of the apo-enzyme (see Tab. S20 and S22). Distances between both $\mathrm{Zn}^{2+}$ ions and their coordinating residues are comparable to the values in the crystal structure of the apo enzyme (see Tab. S20 and S22). The major differences is the increased distance between $\mathrm{Zn} 1$ and carboxylic oxygen of Asp139 in the

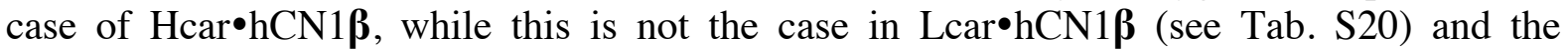
decreasing distance between bridging moiety and both $\mathrm{Zn}^{2+}$ ions in both adducts, compared to the crystal structure (see Tab. S20).

Even though average lengths of $d_{1}$ and $d_{2}$ are longer in Lcar than in Hcar $\left(d_{1}=5.5 \pm 0.4 \AA\right.$ and $d_{2}=5.0 \pm 0.3$ in Lcar $\bullet$ hCN1 $1 \beta$ and $d_{1}=4.1 \pm 0.3 \AA$ and $d_{2}=4.5 \pm 0.2$ in Hcar $\bullet$ hCN1 $\beta$ ), Lcar is again better orientated inside the active site for the hydrolysis reaction. This can be attributed to the fact that Hcar forms strong hydrogen bond with Glh173. According to the proposed mechanisms ${ }^{10,11}$ the proton on Glh173 should instead be interacting with NH group of the ligands peptide bond, which is in this case hampered (see Fig. S11).

\section{Docking of Lcar and Hcar to the hCN1 dimer.}

Each ligand features two distinct docking binding poses, with population of $50 \%$ and $37 \%$ for Lcar (LBP1 and LBP2) and 68\% and 16\% for Hcar (HBP1, HBP2). The peptide bond in LBP2 and HBP2 has identical orientation to the one obtained from the monomer-based simulations (see Fig. S12 and Tab. S18). In the other binding pose (LBP1 and HBP1), which is by far that with the higher population, the peptide oxygen atom forms a hydrogen bond 
with His235 from the other monomer and therefore it does not coordinate the $\mathrm{Zn}^{2+}$ ion as in the monomer (see Fig. S12 and Tab. S18). Hence, LBP1 and HBP1 are most likely populated poses. Therefore, they were selected for further investigation using QM/MM simulations. 
Table S1. Reported $\mathrm{K}_{\mathrm{m}}$ and $\mathrm{k}_{\mathrm{cat}}$ values of a variety of hCN1 substrates. ${ }^{a}$

\begin{tabular}{|l|l|l|l|}
\hline Substrate & $\mathrm{K}_{\mathrm{m}}[\mathrm{mM}]$ & $\mathrm{k}_{\mathrm{cat}}\left[\mathrm{s}^{-1}\right]$ & $\mathrm{k}_{\mathrm{cat}} / \mathrm{K}_{\mathrm{m}}\left[\mathrm{mM}^{-1} \mathrm{~s}^{-1}\right]$ \\
\hline Lcar $^{b}$ & 1.27 & 10.6 & 8.6 \\
\hline Lcar $^{c}$ & $8.0 \pm 0.6$ & - & - \\
\hline Hcar $^{b}$ & 0.2 & 0.2 & 1.1 \\
\hline anserine $^{c}$ & $8.0 \pm 0.6$ & - & - \\
\hline ophidine $^{c}$ & $8.0 \pm 0.6$ & - & - \\
\hline
\end{tabular}

${ }^{a}$ Dash stands if corresponding data is not reported.

${ }^{b} \mathrm{pH} 7.0$ and $30^{\circ} \mathrm{C}$; data from ref. ${ }^{12}$

${ }^{c} \mathrm{pH} 8.5$ and $36^{\circ} \mathrm{C}$; data from ref. ${ }^{13}$ 
Table S2. Bond lengths (in $\AA$ ) for tautomers A and B. Atom types are gaff atom types ${ }^{14}$ (see also Tab. S4).

\begin{tabular}{|c|c|c|c|}
\hline \multicolumn{2}{|c|}{$\mathbf{A}$} & \multicolumn{2}{|c|}{ B } \\
\hline bond & $\begin{array}{l}\text { bond } \\
\text { length }\end{array}$ & bond & $\begin{array}{l}\text { bond } \\
\text { length }\end{array}$ \\
\hline hn-n4 & 1.03 & hn-n4 & 1.03 \\
\hline n4-c3 & 1.54 & n4-c3 & 1.53 \\
\hline hx-c3 & 1.09 & hx-c3 & 1.09 \\
\hline c3-c3 & 1.53 & c3-c3 & 1.53 \\
\hline hc-c3 & 1.10 & hc-c3 & 1.10 \\
\hline c3-c & 1.58 & c3-c & 1.58 \\
\hline $\mathrm{c}-\mathrm{O}$ & 1.25 & c-o & 1.25 \\
\hline c-n & 1.32 & c-n & 1.32 \\
\hline n-hn & 1.05 & n-hn & 1.06 \\
\hline$n-c 3$ & 1.46 & $n-c 3$ & 1.46 \\
\hline c3-h1 & 1.10 & c3-h1 & 1.10 \\
\hline c3-cd & 1.50 & c3-cd & 1.50 \\
\hline cd-cc & 1.38 & cd-cc & 1.38 \\
\hline na-hn & 1.04 & na-hn & 1.01 \\
\hline cc-h4 & 1.08 & cc-h4 & 1.08 \\
\hline cc-nc & 1.38 & cc-na & 1.37 \\
\hline nc-cd & 1.32 & nd-cc & 1.32 \\
\hline cd-na & 1.37 & cd-nd & 1.39 \\
\hline h5-cd & 1.08 & cc-h5 & 1.08 \\
\hline
\end{tabular}


Table S3. Angle values (in ${ }^{\circ}$ ) for tautomers $\mathbf{A}$ and $\mathbf{B}$. Atom types are gaff atom types ${ }^{14}$ (see also Tab. S4).

\begin{tabular}{|c|c|c|c|}
\hline \multicolumn{2}{|c|}{$\mathbf{A}$} & \multicolumn{2}{|c|}{ B } \\
\hline angle & $\begin{array}{l}\text { angle } \\
\text { value }\end{array}$ & angle & $\begin{array}{l}\text { angle } \\
\text { value }\end{array}$ \\
\hline hn-n4-hn & 107.9 & hn-n4-hn & 107.9 \\
\hline hn-n4-c3 & 111.0 & hn-n4-c3 & 111.0 \\
\hline n4-c3-hx & 106.8 & n4-c3-hx & 106.8 \\
\hline $\mathrm{n} 4-\mathrm{c} 3-\mathrm{c} 3$ & 111.3 & $\mathrm{n} 4-\mathrm{c} 3-\mathrm{c} 3$ & 111.3 \\
\hline hx-c3-hx & 109.9 & $h x-c 3-h x$ & 110.1 \\
\hline hx-c3-c3 & 110.9 & $h x-c 3-c 3$ & 110.8 \\
\hline c3-c3-hc & 109.8 & c3-c3-hc & 109.9 \\
\hline hc-c3-hc & 107.7 & hc-c3-hc & 107.6 \\
\hline c3-c3-c & 110.4 & c3-c3-c & 110.5 \\
\hline hc-c3-c & 109.6 & hc-c3-c & 109.5 \\
\hline c3-c-o & 116.4 & c3-c-o & 116.1 \\
\hline c3-c-n & 113.8 & c3-c-n & 113.5 \\
\hline o-c-n & 128.6 & o-c-n & 129.2 \\
\hline c-n-hn & 126.0 & c-n-hn & 126.8 \\
\hline c-n-c3 & 127.8 & c-n-c3 & 128.0 \\
\hline hn-n-c3 & 105.0 & hn-n-c3 & 103.4 \\
\hline n-c3-c & 102.9 & n-c3-c & 102.5 \\
\hline n-c3-h1 & 109.5 & n-c3-h1 & 109.9 \\
\hline$n-c 3-c 3$ & 112.1 & $n-c 3-c 3$ & 111.0 \\
\hline h1-c3-c3 & 108.6 & h1-c3-c3 & 109.1 \\
\hline h1-c3-c & 109.0 & h1-c3-c & 109.3 \\
\hline O-c-o & 128.2 & O-c-o & 129.1 \\
\hline hc-c3-cd & 109.3 & hc-c3-cd & 108.5 \\
\hline c3-cd-cc & 132.5 & c3-cd-cc & 129.6 \\
\hline c3-c3-cd & 114.5 & c3-c3-cd & 116.0 \\
\hline c3-cd-na & 122.4 & c3-cd-nd & 120.2 \\
\hline cd-cc-nc & 110.9 & cd-cc-na & 105.3 \\
\hline
\end{tabular}




\begin{tabular}{|l|c|l|c|}
\hline cd-cc-h4 & 127.6 & cd-cc-h4 & 129.4 \\
\hline h4-cc-nc & 121.5 & h4-cc-na & 125.3 \\
\hline cc-nc-cd & 104.5 & cc-na-cc & 107.4 \\
\hline cd-na-cd & 107.1 & cd-nd-cc & 105.5 \\
\hline nc-cd-h5 & 125.3 & na-cc-h5 & 122.4 \\
\hline cd-na-hn & 126.3 & cc-na-hn & 126.3 \\
\hline nc-cd-na & 112.4 & na-cc-nd & 111.6 \\
\hline h5-cd-na & 122.3 & h5-cc-nd & 126.0 \\
\hline na-cd-cc & 105.1 & nd-cd-cc & 110.2 \\
\hline
\end{tabular}


Table S4. Partial atom charges of atoms in A and $\mathbf{B}$ together with legend of labeling of atoms in $\mathbf{A}$ and $\mathbf{B}$. Atom types are gaff atom types. ${ }^{14}$

\begin{tabular}{|c|c|c|c|c|c|}
\hline $\mathbf{A}$ & & & B & & \\
\hline atom type & atom label & charge & atom type & atom label & charge \\
\hline $\mathrm{nc}$ & $\mathrm{N}_{\varepsilon}$ & -0.557 & na & $\mathrm{N}_{\varepsilon}$ & -0.291 \\
\hline na & $\mathrm{N}_{\delta}$ & -0.250 & nd & $\mathrm{N}_{\delta}$ & -0.583 \\
\hline $\mathrm{hn}$ & $\mathrm{H}\left(\right.$ on $\left.\mathrm{N}_{\delta}\right)$ & 0.266 & hn & $\mathrm{H}\left(\right.$ on $\left.\mathrm{N}_{\varepsilon}\right)$ & 0.300 \\
\hline $\mathrm{cd}$ & $\mathrm{C}_{\varepsilon}$ & 0.232 & $\mathrm{cc}$ & $\overline{\mathrm{C}_{\varepsilon}}$ & 0.229 \\
\hline h5 & $\mathrm{H}\left(\right.$ on $\left.\mathrm{C}_{\varepsilon}\right)$ & 0.057 & h5 & $\mathrm{H}\left(\right.$ on $\left.\mathrm{C}_{\varepsilon}\right)$ & 0.055 \\
\hline $\mathrm{cc}$ & $\mathrm{C}_{\delta}$ & 0.080 & $\mathrm{cc}$ & $\mathrm{C}_{\delta}$ & -0.266 \\
\hline h4 & $\mathrm{H}\left(\right.$ on $\left.\mathrm{C}_{\delta}\right)$ & 0.062 & $\mathrm{~h} 4$ & $\mathrm{H}\left(\right.$ on $\left.\mathrm{C}_{\delta}\right)$ & 0.194 \\
\hline $\mathrm{cd}$ & $\mathrm{C}_{\gamma}$ & -0.030 & $\mathrm{~cd}$ & $\overline{C_{\gamma}}$ & 0.369 \\
\hline c3 & $\overline{C_{\beta}}$ & -0.072 & c3 & $\overline{C_{\beta}}$ & -0.122 \\
\hline hc & $\mathrm{H}\left(\right.$ on $\left.\mathrm{C}_{\beta}\right)$ & 0.029 & $\mathrm{hc}$ & $\mathrm{H}\left(\right.$ on $\left.\mathrm{C}_{\beta}\right)$ & 0.038 \\
\hline $\mathrm{c3}$ & $\overline{\mathrm{C}_{\alpha}}$ & 0.327 & $\mathrm{c3}$ & $\mathrm{C}_{\alpha}$ & 0.177 \\
\hline $\mathrm{c}$ & $\begin{array}{l}\mathrm{C} \\
\text { (carboxyl) }\end{array}$ & 0.605 & $\mathrm{c}$ & $\begin{array}{l}\mathrm{C} \\
\text { (carboxyl) }\end{array}$ & 0.639 \\
\hline $\mathrm{o}$ & $\begin{array}{l}\mathrm{O} \\
\text { (carboxyl) }\end{array}$ & -0.624 & $\mathrm{o}$ & $\begin{array}{l}\mathrm{O} \\
\text { (carboxyl) }\end{array}$ & -0.650 \\
\hline h1 & $\mathrm{H}\left(\right.$ on $\left.\mathrm{C}_{\alpha}\right)$ & -0.039 & h1 & $\mathrm{H}\left(\right.$ on $\left.\mathrm{C}_{\alpha}\right)$ & -0.024 \\
\hline $\mathrm{n}$ & $\mathrm{N}$ (amide) & -0.628 & $\mathrm{n}$ & $\mathrm{N}$ (amide) & -0.501 \\
\hline $\mathrm{hn}$ & $\mathrm{H}$ (amide) & 0.281 & $\mathrm{hn}$ & $\mathrm{H}$ (amide) & 0.261 \\
\hline $\mathrm{c}$ & $\begin{array}{l}\mathrm{C} \\
\text { (carbonyl) }\end{array}$ & 0.660 & $\mathrm{c}$ & $\begin{array}{l}\mathrm{C} \\
\text { (carbonyl) }\end{array}$ & 0.599 \\
\hline $\mathrm{o}$ & $\begin{array}{l}\mathrm{O} \\
\text { (carbonyl) }\end{array}$ & -0.563 & o & $\begin{array}{l}\mathrm{O} \\
\text { (carbonyl) }\end{array}$ & -0.553 \\
\hline c3 & $\overline{\mathrm{C}_{\alpha^{\prime}}}$ & -0.272 & c3 & $\overline{C_{\alpha}}$ & -0.271 \\
\hline hc & $\mathrm{H}\left(\right.$ on $\mathrm{C}_{\alpha}$ ) & 0.088 & hc & $\mathrm{H}\left(\right.$ on $\mathrm{C}_{\alpha}$ ) & 0.079 \\
\hline c3 & $\overline{C_{\beta}}$, & 0.173 & c3 & $\mathrm{C}_{\beta}$, & 0.233 \\
\hline $\mathrm{hx}$ & $\mathrm{H}\left(\right.$ on $\mathrm{C}_{\beta}$ ) & 0.060 & $\mathrm{hx}$ & $\mathrm{H}\left(\right.$ on $\mathrm{C}_{\beta}$ ) & 0.041 \\
\hline $\mathrm{n} 4$ & $\mathrm{~N}$ (amine) & -0.427 & $\mathrm{n} 4$ & $\mathrm{~N}$ (amine) & -0.441 \\
\hline $\mathrm{hn}$ & $\mathrm{H}$ (amine) & 0.328 & $\mathrm{hn}$ & $\mathrm{H}$ (amine) & 0.328 \\
\hline
\end{tabular}


Table S5. Cosine content of the first four principal components for $\mathbf{A}$ and $\mathbf{B}$ for the two independent simulations of each tautomer and for one simulation of $\mathbf{C}$ and $\mathbf{D}$.

\begin{tabular}{|l|c|c|c|c|c|c|}
\hline \multirow{2}{*}{$\begin{array}{c}\text { principal } \\
\text { component }\end{array}$} & \multicolumn{5}{|c|}{ cosine content of principal component } \\
\cline { 2 - 7 } & \multicolumn{2}{|c|}{ A } & \multicolumn{2}{c|}{ B } & C & D \\
\hline 1 & 0.0011 & 0.0019 & $7.3 * 10^{-5}$ & $1.5^{*} 10^{-5}$ & 0.0007 & 0.0017 \\
\hline 2 & $9.8^{*} 10^{-6}$ & 0.0020 & 0.0005 & $4.6^{*} 10^{-5}$ & $6.7 * 10^{-5}$ & $2.4^{*} 10^{-7}$ \\
\hline 3 & 0.0001 & $4.8^{*} 10^{-6}$ & 0.0002 & $1.4^{*} 10^{-5}$ & 0.0003 & $2.7 * 10^{-5}$ \\
\hline 4 & $1.4^{*} 10^{-6}$ & 0.0008 & $6.9^{*} 10^{-5}$ & $2.6^{*} 10^{-6}$ & 0.0006 & $3.4^{*} 10^{-6}$ \\
\hline
\end{tabular}

Table S6. Protonation states of histidine residues and of Glu173. The acidic amino acids other than Glu173 are negatively charged.

\begin{tabular}{|l|l|}
\hline \multicolumn{1}{|c|}{ Residue number } & \multicolumn{1}{c|}{ Protonation state } \\
\hline $106,314,324,367,452$ & HID \\
\hline $235,244,263,388,481$ & HIE \\
\hline $39,288,354,436$ & HIP \\
\hline 173 & GLH \\
\hline
\end{tabular}

Table S7. Shift of the water molecule (in $\AA$ ) after docking of Lcar and Hcar to the hCN1 active site. Results from six docking with highest score for each ligand are reported.

\begin{tabular}{|l|c|c|c|c|c|c|c|}
\hline $\begin{array}{c}\text { Docking } \\
\text { number }\end{array}$ & 1 & 2 & 3 & 4 & 5 & 6 & $\begin{array}{c}\text { Average } \\
\text { shift }\end{array}$ \\
\hline Lcar & 1.97 & 1.62 & 1.58 & 1.84 & 1.45 & 1.59 & 1.68 \\
\hline Hcar & 1.46 & 1.54 & 1.72 & 1.53 & 1.59 & 1.63 & 1.58 \\
\hline
\end{tabular}


Table S8. Lcar's hydrogen bond functionalities form hydrogen bonds with water molecules (similarly to what was found in ref. ${ }^{4}$ ). The table shows the average and maximum water coordination numbers of hydrogen bond acceptors/donors for $\mathbf{A}$ and $\mathbf{B}$. For both tautomers, all hydrogen bond acceptors and donors form hydrogen bond with water molecules with around $80 \%$ probability also consistent with their similar size of solvent accessible surface (see Fig. S4).

\begin{tabular}{|c|c|c|c|c|}
\hline \multirow[b]{2}{*}{$\begin{array}{l}\text { hydrogen bond } \\
\text { acceptor/donor }\end{array}$} & \multicolumn{2}{|c|}{$\mathbf{A}$} & \multicolumn{2}{|c|}{ B } \\
\hline & $\begin{array}{l}\text { average water } \\
\text { coordination } \\
\text { number }\end{array}$ & $\begin{array}{c}\text { maximum water } \\
\text { coordination } \\
\text { number }\end{array}$ & $\begin{array}{c}\text { average water } \\
\text { coordination } \\
\text { number }\end{array}$ & $\begin{array}{c}\text { maximum water } \\
\text { coordination } \\
\text { number }\end{array}$ \\
\hline $\mathrm{H} \delta$ & $0.81 \pm 0.33$ & 1.07 & / & I \\
\hline $\mathrm{H \varepsilon}$ & I & / & $0.83 \pm 0.32$ & 1.05 \\
\hline $\mathrm{H}$ (amide) & $0.79 \pm 0.36$ & 1.11 & $0.79 \pm 0.36$ & 1.11 \\
\hline $\begin{array}{l}\mathrm{H} \text { (primary } \\
\text { amine) }\end{array}$ & $0.85 \pm 0.32$ & 1.56 & $0.85 \pm 0.32$ & 1.67 \\
\hline $\mathrm{H} \alpha$ & $0.78 \pm 0.37$ & 1.10 & $0.78 \pm 0.37$ & 1.12 \\
\hline $\mathrm{H} \varepsilon($ on $\mathrm{C} \varepsilon$ ) & $0.82 \pm 0.36$ & 1.15 & $0.82 \pm 0.36$ & 1.19 \\
\hline O (carboxyl) & $0.84 \pm 0.33$ & 1.89 & $0.85 \pm 0.33$ & 2.02 \\
\hline O (carbonyl) & $0.81 \pm 0.34$ & 1.27 & $0.81 \pm 0.34$ & 1.37 \\
\hline No & $0.79 \pm 0.37$ & 1.04 & $0.81 \pm 0.34$ & 1.08 \\
\hline $\mathrm{N \varepsilon}$ & $0.82 \pm 0.33$ & 1.24 & $0.80 \pm 0.37$ & 1.08 \\
\hline
\end{tabular}


Table S9. Calculated ${ }^{13} \mathrm{C}$ chemical shifts of both zwitterionic conformers of Lcar in comparison with experimental values. ${ }^{a}$ For labeling, see Chart 1 . The highest difference in tetramethylsilane (TMS) between experimental and calculated values are at C $\alpha$ ' (experimental values range from 33.7 to $36.1 \mathrm{ppm}$ compared to the calculated value of 47.0 ppm), while in sodium [2,2,3,3-2H4]-3-trimethylsilylpropanoate the highest difference is at $\mathrm{C} \beta$ ' (experimental is $32.3 \mathrm{ppm}$ compared to the calculated value of $49.6 \mathrm{ppm}$ ).

\begin{tabular}{|l|c|c|c|c|c|}
\hline $\begin{array}{c}\text { carbon } \\
\text { atom }\end{array}$ & $\begin{array}{c}\text { Friedrich } \\
\text { [ppm] } \\
(\mathrm{T}=294 \mathrm{~K})\end{array}$ & $\begin{array}{c}\text { Branham } \\
\text { [ppm] } \\
\text { (T not } \\
\text { reported })\end{array}$ & $\begin{array}{c}\text { Gaggelli } \\
\text { [ppm] } \\
(\mathrm{T}=295 \mathrm{~K})\end{array}$ & $\begin{array}{c}\text { calculated } \\
\text { chemical } \\
\text { shift }^{\mathrm{b}} \text { [ppm] }\end{array}$ & assignments \\
\hline $\mathrm{CO}_{2}{ }^{-}$ & 179.1 & 177.9 & 177.9 & $180.2(182.0)$ & carboxylate \\
\hline $\mathrm{C}(\mathrm{O}) \mathrm{NH}$ & 172.9 & 171.7 & 171.6 & $162.8(164.7)$ & amide carbonyl \\
\hline $\mathrm{C} \varepsilon$ & 137.0 & 135.9 & 135.7 & $135.5(137.4)$ & imine carbon \\
\hline $\mathrm{C} \gamma$ & 134.5 & 117.6 & 133.2 & $144.6(146.5)$ & ring carbon atom \\
\hline $\mathrm{C} \delta$ & 118.7 & 133.2 & 117.4 & $121.6(123.5)$ & ring methine \\
carbon
\end{tabular}

${ }^{a}$ Friedrich ${ }^{15}$ and Branham ${ }^{16}$ used TMS as a reference compound, while Gaggelli ${ }^{17}$ used sodium [2,2,3,3-2H4]-3-trimethylsilylpropanoate.

${ }^{\mathrm{b}}$ Calculated chemical shifts are reported with respect to the TMS and sodium [2,2,3,3-2H4]3-trimethylsilylpropanoate (in parentheses). 
Table S10. Not-weighted calculated ${ }^{13} \mathrm{C}$ chemical shifts of $\mathbf{1 A - 4 A}$ and $\mathbf{1 B}-\mathbf{4 B}$ conformers of Lcar. Chemical shifts were scaled with respect to the chemical shift of ${ }^{13} \mathrm{C}$ TMS.

\begin{tabular}{|c|c|c|c|c|c|c|c|c|c|}
\hline$\hat{F}$ & $\begin{array}{l}m \\
\infty \\
\infty\end{array}$ & $\begin{array}{l}0 \\
\dot{0} \\
0\end{array}$ & $\begin{array}{l}0 \\
\infty \\
\infty \\
\end{array}$ & 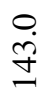 & $\stackrel{0}{\stackrel{\Delta}{\beth}}$ & $\begin{array}{l}\infty \\
\dot{f}\end{array}$ & $\stackrel{\circ}{\stackrel{m}{m}}$ & $\stackrel{\vartheta}{\dot{\gamma}}$ & ले \\
\hline लै & $\stackrel{+}{\dot{\infty}}$ & $\underset{\tilde{\sigma}}{\tilde{\sigma}}$ & $\begin{array}{l}0 \\
\ddot{n} \\
\end{array}$ & 苢 & $\begin{array}{l}\dot{0} \\
\stackrel{\varrho}{=}\end{array}$ & $\stackrel{9}{\infty}$ & $\frac{0}{i n}$ & $\stackrel{\circ}{+}$ & $\stackrel{\vec{n}}{m}$ \\
\hline สิ & $\stackrel{m}{\infty}$ & $\overrightarrow{0}$ & $\stackrel{\circ}{\stackrel{9}{2}}$ & $\stackrel{0}{\stackrel{0}{n}}$ & $\begin{array}{l}0 \\
\stackrel{1}{\Xi}\end{array}$ & $\begin{array}{l}0 \\
\infty \\
\infty\end{array}$ & $\vec{n}$ & $\frac{n}{n}$ & $\stackrel{m}{q}$ \\
\hline$\vartheta$ & $\underset{\infty}{\infty}$ & $\begin{array}{l}0 \\
\text { î } \\
\underline{-}\end{array}$ & 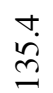 & $\begin{array}{l}0 \\
\dot{J}\end{array}$ & $\stackrel{\infty}{\stackrel{\Xi}{I}}$ & $\begin{array}{l}0 \\
\infty \\
\infty\end{array}$ & $\underset{+}{+}$ & $\frac{?}{f}$ & $\overrightarrow{\text { ले }}$ \\
\hline$\Varangle$ & 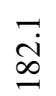 & $\begin{array}{l}0 \\
\stackrel{0}{\Xi}\end{array}$ & $\begin{array}{l}\infty \\
\infty \\
\infty \\
\end{array}$ & 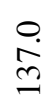 & $\stackrel{\vec{f}}{\sigma}$ & గి & $\stackrel{\circ}{\stackrel{0}{n}}$ & rִ & $\stackrel{n}{n}$ \\
\hline$\overleftrightarrow{m}$ & $\begin{array}{l}m \\
\infty \\
\infty\end{array}$ & 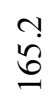 & $\stackrel{n}{\sharp}$ & $\begin{array}{l}0 \\
\stackrel{2}{2}\end{array}$ & $\stackrel{\vec{n}}{\ddot{q}}$ & $\begin{array}{l}\infty \\
\infty \\
\infty\end{array}$ & $\stackrel{\check{f}}{\dot{f}}$ & $\stackrel{\dddot{q}}{\stackrel{\gamma}{\gamma}}$ & $\stackrel{m}{m}$ \\
\hline 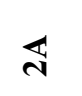 & ֶี & $\stackrel{n}{n}$ & 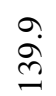 & $\begin{array}{l}0 \\
\dot{d} \\
\ddot{g}\end{array}$ & 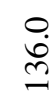 & $\begin{array}{l}0 \\
\dot{0} \\
0\end{array}$ & ?n. & $\begin{array}{l}\infty \\
\stackrel{n}{ }\end{array}$ & $\underset{\infty}{\infty}$ \\
\hline$\unlhd$ & $\stackrel{m}{\stackrel{m}{0}}$ & ㄹ. & $\stackrel{\tilde{I}}{\mathrm{I}}$ & $\stackrel{\circ}{\stackrel{+}{J}}$ & $\stackrel{\sigma}{\underline{m}}$ & $\overline{8}$ & $\begin{array}{l}\infty \\
\dot{q}\end{array}$ & $\begin{array}{l}\infty \\
\dot{\sigma} \\
\dot{q}\end{array}$ & $\underset{\text { m. }}{0}$ \\
\hline 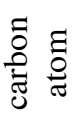 & O & $\underset{\mathrm{U}}{\stackrel{\mathrm{J}}{\mathrm{z}}}$ & U & U & U & U & $\ddot{U}$ & $\ddot{u}$ & uे \\
\hline
\end{tabular}


Table S11. Calculated and experimentally observed ${ }^{1} \mathrm{H}$ chemical shifts of Lcar. ${ }^{\text {a }}$ For labeling, see Chart 1 . The highest difference between experimental and calculated values is in both cases is at $\mathrm{H} \beta$ '. In TMS experimental values range from 2.60 to $3.10 \mathrm{ppm}$ compared to the calculated value of $3.68 \mathrm{ppm}$ and in sodium [2,2,3,3-2H4]-3-trimethylsilylpropanoate the experimental value is 1.88 compared to the calculated one of $3.72 \mathrm{ppm}$.

\begin{tabular}{|l|c|c|c|c|c|c|}
\hline $\begin{array}{c}\text { hydrogen } \\
\text { atom }\end{array}$ & $\begin{array}{c}\text { Branham } \\
{[\mathrm{ppm}]} \\
(\mathrm{T} \text { not } \\
\text { reported })\end{array}$ & $\begin{array}{c}\text { Zhang }^{4} \\
{[\mathrm{ppm}]} \\
(\mathrm{T}=298 \mathrm{~K})\end{array}$ & $\begin{array}{c}\text { Friedrich }^{15} \\
{[\mathrm{ppm}]} \\
(\mathrm{T}=294 \mathrm{~K})\end{array}$ & $\begin{array}{c}\text { Gaggelli }^{17} \\
{[\mathrm{ppm}]} \\
(\mathrm{T}=295 \mathrm{~K})\end{array}$ & $\begin{array}{c}\text { calculated } \\
\text { chemical } \\
\text { shifts }^{\mathrm{b}}[\mathrm{ppm}]\end{array}$ & assignments \\
\hline $\mathrm{H} \varepsilon$ & 7.66 & 7.60 & 7.38 & 6.93 & $7.72(7.76)$ & imine hydrogen \\
\hline $\mathrm{H} \delta$ & 6.89 & 6.83 & 6.63 & 6.17 & $7.10(7.14)$ & ring methine \\
\hline $\mathrm{H} \beta_{\mathrm{R}}, \mathrm{H} \beta_{\mathrm{S}}$ & $3.08 ; 2.92$ & $3.01 ; 2.85^{\mathrm{c}}$ & $2.65 ; 2.82$ & $2.37 ; 2.21^{\mathrm{c}}$ & $\begin{array}{c}2.74 ; 3.38 \\
(2.79 ; 3.42)\end{array}$ & $\beta$-His-methylene \\
\hline $\mathrm{H \alpha}$ & 4.40 & 4.34 & 4.15 & 3.71 & $3.64(3.68)$ & $\alpha$-His-methine \\
\hline $\mathrm{NH}$ & 7.89 & - & - & - & $7.42(7.46)$ & amide N-H \\
\hline $\mathrm{H \alpha} \alpha^{\prime}\left(\mathrm{CH}_{2}\right)$ & 3.16 & 2.53 & 2.35 & 2.44 & $2.64(2.69)$ & $\alpha$-Ala-methylene \\
\hline $\mathrm{H} \beta{ }^{\prime}\left(\mathrm{CH}_{2}\right)$ & 2.60 & 3.10 & 2.90 & 1.88 & $3.68(3.72)$ & $\beta$-Ala-methylene \\
\hline
\end{tabular}

${ }^{a}$ Friedrich ${ }^{15}$ and Branham $^{16}$ used TMS as a reference compound, while Gaggelli ${ }^{17}$ used sodium [2,2,3,3-2H4]-3-trimethylsilylpropanoate.

${ }^{\mathrm{b}}$ Calculated chemical shifts are reported with respect to the TMS and sodium [2,2,3,3-2H4]3-trimethylsilylpropanoate (in parentheses).

${ }^{c}$ In the reference it is not mentioned which shift belongs to which hydrogen atom. 
Table S12. Not-weighted calculated ${ }^{1} \mathrm{H}$ chemical shifts of $\mathbf{1 A - 4 A}$ and $\mathbf{1 B}-\mathbf{4 B}$ conformers of Lcar. Chemical shifts were scaled with respect to the chemical shift of ${ }^{1} \mathrm{H}$ TMS.

\begin{tabular}{|c|c|c|c|c|c|c|c|}
\hline$\vartheta$ & $\stackrel{0}{\pi}$ & $\stackrel{\text { }}{\circ}$ & 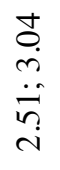 & 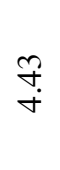 & $\underset{\sim}{\stackrel{\infty}{r}}$ & $\begin{array}{l}\stackrel{\circ}{n} \\
\text { r. }\end{array}$ & $\stackrel{\infty}{\tilde{n}}$ \\
\hline లै & $\stackrel{n}{r}$ & જิ & $\begin{array}{l}\vec{\forall} \\
\ddot{\sim} \\
\ddot{\sigma}\end{array}$ & $\begin{array}{l}\stackrel{\circ}{\circ} \\
\text { r. }\end{array}$ & $\bar{n}$ & $\underset{i}{\stackrel{J}{i}}$ & $\stackrel{n}{\hat{m}}$ \\
\hline สิ & $\stackrel{尺}{i}$ & $\begin{array}{l}\stackrel{2}{r} \\
r\end{array}$ & $\begin{array}{l}\hat{N} \\
\ddot{n} \\
\ddot{\infty} \\
\dot{d}\end{array}$ & $\stackrel{m}{\stackrel{n}{+}}$ & $\stackrel{n}{n}$ & $\stackrel{\infty}{\stackrel{\infty}{i}}$ & $\stackrel{\partial}{\vec{m}}$ \\
\hline$\vartheta$ & $\stackrel{+}{\stackrel{丶}{r}}$ & $\underset{ }{\stackrel{0}{r}}$ & $\begin{array}{l}\stackrel{q}{+} \\
\dot{r} \\
\ddot{n} \\
\dot{i}\end{array}$ & $\stackrel{n}{n}$ & $\stackrel{\grave{r}}{r}$ & $\begin{array}{l}n \\
i \\
i\end{array}$ & $\stackrel{\partial}{\vec{m}}$ \\
\hline$\S$ & $\stackrel{n}{r}$ & $\stackrel{\infty}{\underset{\pi}{\pi}}$ & $\begin{array}{l}\sigma \\
\dot{\sigma} \\
\ddot{a} \\
\ddot{\sigma} \\
\dot{m}\end{array}$ & $\underset{+}{\stackrel{f}{f}}$ & लై & $\stackrel{n}{i}$ & nे \\
\hline$\overleftrightarrow{m}$ & $\stackrel{\infty}{\stackrel{\infty}{r}}$ & $\underset{r}{\vec{r}}$ & 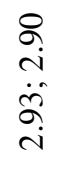 & $\stackrel{\infty}{\underset{+}{+}}$ & $\bar{\infty}$ & $\underset{i}{\stackrel{i}{i}}$ & $\underset{\text { iे. }}{\text { in }}$ \\
\hline$\overleftrightarrow{\sim}$ & $\stackrel{n}{r}$ & $\underset{r}{\stackrel{d}{r}}$ & $\begin{array}{l}\vec{\infty} \\
\dot{r} \\
\dot{\sigma} \\
\vec{i}\end{array}$ & $\underline{\text { in }}$ & $\bar{\sigma}_{\infty}$ & $\underset{i}{\stackrel{8}{i}}$ & $\frac{\infty}{m}$ \\
\hline$\Xi$ & $\stackrel{?}{r}$ & 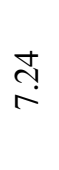 & $\begin{array}{l}\stackrel{\sim}{n} \\
\grave{\infty} \\
\dot{\infty} \\
i\end{array}$ & 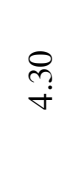 & ర్ర. & $\begin{array}{l}\hat{\sigma} \\
i\end{array}$ & $\underset{\sim}{\vec{r}}$ \\
\hline 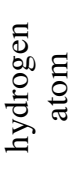 & $\stackrel{\omega}{\Psi}$ & $\stackrel{0}{\mathcal{I}}$ & $\begin{array}{l}\frac{n}{\tilde{I}} \\
\stackrel{\tilde{n}}{\tilde{I}}\end{array}$ & ర્વ & $\overline{\mathbf{Z}}$ & 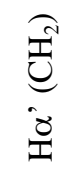 & 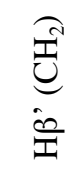 \\
\hline
\end{tabular}


Table S13. Calculated and experimentally observed ${ }^{1} \mathrm{H}$ chemical shifts of Hcar. For labeling, see Chart 1 . The highest difference between experimental and calculated values are at aminoterminal group (experimental values is $6.40 \mathrm{ppm}$ compared to the calculated one of 4.65 ppm).

\begin{tabular}{|l|c|c|c|}
\hline $\begin{array}{c}\text { hydrogen } \\
\text { atom }\end{array}$ & $\begin{array}{c}\text { Govindaraju } \\
\text { [ppm] } \\
(\mathrm{T}=310 \mathrm{~K})\end{array}$ & $\begin{array}{c}\text { calculated } \\
\text { chemical } \\
\text { shifts } \\
\text { [ppm] }\end{array}$ & assignments \\
\hline $\mathrm{H} \varepsilon$ & 7.08 & 7.27 & imine hydrogen \\
\hline $\mathrm{H} \delta$ & 8.08 & 6.88 & ring methine \\
\hline $\mathrm{H} \beta_{\mathrm{R}}, \mathrm{H} \beta_{\mathrm{S}}$ & $3.00 ; 3.19^{\mathrm{a}}$ & $2.82 ; 2.72$ & $\beta$-His-methylene \\
\hline $\mathrm{H} \alpha$ & 4.47 & 4.58 & $\begin{array}{c}\alpha \text {-His-methine } \\
\mathrm{H} \alpha^{\prime}\left(\mathrm{CH}_{2}\right)\end{array}$ \\
\hline $\mathrm{H} \beta^{\prime}\left(\mathrm{CH}_{2}\right)$ & 2.96 & 2.20 & $\begin{array}{c}\alpha \text {-amino-butyryl- } \\
\text { methylene }\end{array}$ \\
\hline $\mathrm{H} \gamma^{\prime}\left(\mathrm{CH}_{2}\right)$ & 2.89 & 1.85 & $\begin{array}{c}\beta \text {-amino-butyryl- } \\
\text { methylene }\end{array}$ \\
\hline $\mathrm{NH}$ & 6.40 & 4.65 & $\begin{array}{c}\gamma \text {-amino-butyryl- } \\
\text { methylene }\end{array}$ \\
\hline
\end{tabular}

${ }^{\mathrm{a}}$ In the reference it is not mentioned which shift belongs to which hydrogen atom. 
Table S14. Not-weighted calculated ${ }^{1} \mathrm{H}$ chemical shifts of $\mathbf{1 C}-\mathbf{4 C}$ and $\mathbf{1 D}-\mathbf{4 D}$ conformers of Lcar. Chemical shifts were scaled with respect to the chemical shift of ${ }^{11} \mathrm{H}$ DSS.

\begin{tabular}{|c|c|c|c|c|c|c|c|c|}
\hline$f$ & $\stackrel{n}{r}$ & $\stackrel{\infty}{\stackrel{\circ}{r}}$ & 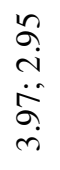 & $\stackrel{\overbrace{}}{\tilde{r}}$ & 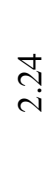 & $\begin{array}{l}\text { ণ্ } \\
\text { in }\end{array}$ & $\underset{i}{\stackrel{ \pm}{n}}$ & $\underset{\sigma}{\sigma}$ \\
\hline ले & 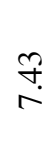 & $\bar{\sigma}$ & 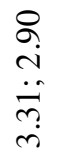 & $\hat{r}$ & $\begin{array}{l}n \\
\tilde{n} \\
n\end{array}$ & $\underset{\sim}{\stackrel{\sim}{c}}$ & $\stackrel{\vec{r}}{\stackrel{\theta}{ }}$ & $\stackrel{n}{r}$ \\
\hline สิ & $\stackrel{\widehat{N}}{r}$ & $\begin{array}{l}\infty \\
\infty \\
0 \\
0\end{array}$ & $\begin{array}{l}\vec{i} \\
i \\
\dot{i} \\
\infty \\
i \\
i\end{array}$ & $\stackrel{\infty}{\stackrel{\infty}{+}}$ & તָ & $\stackrel{\wp}{\infty}$ & $\stackrel{\bar{m}}{\vec{n}}$ & $\begin{array}{l}\mathfrak{6} \\
+\end{array}$ \\
\hline$\vartheta$ & $\underset{\infty}{\infty}$ & nִ & $\begin{array}{l}= \\
\ddot{m} \\
\ddot{n} \\
\dot{n}\end{array}$ & $\underset{\dot{\sigma}}{\tilde{\sigma}}$ & $\stackrel{n}{c}$ & 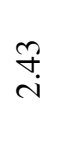 & $\begin{array}{l}\text { Do } \\
\text { ri }\end{array}$ & $\stackrel{゚}{+}$ \\
\hline$\dot{t}$ & $\stackrel{\overbrace{}}{r}$ & $\vec{\sim}$ & $\begin{array}{l}\mathscr{0} \\
\infty \\
i \\
\ddot{\sigma} \\
\dot{m}\end{array}$ & $\stackrel{\mathcal{T}}{7}$ & in & $\begin{array}{c}\text { ণn} \\
\text { in }\end{array}$ & 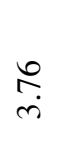 & $\stackrel{゚}{\stackrel{r}{+}}$ \\
\hline Uల & $\underset{\infty}{\infty}$ & $\stackrel{m}{r}$ & $\begin{array}{l}\stackrel{8}{a} \\
i \\
\ddot{\infty} \\
\stackrel{n}{n}\end{array}$ & $\stackrel{\infty}{\stackrel{\infty}{\sim}}$ & $\widehat{\hat{N}}$ & $\underset{i}{\stackrel{\sim}{i}}$ & $\underset{\sim}{\stackrel{8}{\infty}}$ & $\stackrel{\infty}{\stackrel{\infty}{+}}$ \\
\hline U్ & 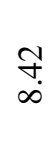 & $\stackrel{7}{\pi}$ & $\begin{array}{l}m \\
\ddot{0} \\
\ddot{n} \\
\ddot{n}\end{array}$ & $\stackrel{\leftrightarrow}{+}$ & $\vec{r}$ & तે & $\stackrel{?}{\stackrel{n}{r}}$ & $\frac{0}{1 n}$ \\
\hline$\underline{U}$ & $\stackrel{n}{r}$ & $\stackrel{\simeq}{\sim}$ & $\begin{array}{l}\infty \\
\dot{i} \\
\ddot{\delta} \\
\dot{\delta} \\
\dot{r}\end{array}$ & $\frac{n}{n}$ & 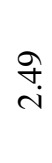 & $\underset{\sim}{\tilde{i}}$ & $\stackrel{\infty}{\stackrel{\infty}{n}}$ & $\underset{+}{\mathbb{t}}$ \\
\hline 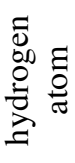 & $\stackrel{\omega}{\Psi}$ & $\stackrel{\infty}{1}$ & $\begin{array}{l}\stackrel{n}{I} \\
\stackrel{\tilde{I}}{\cong}\end{array}$ & $\underset{\mathcal{X}}{\mathcal{X}}$ & 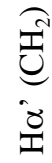 & 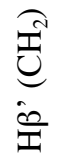 & 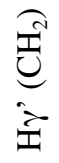 & $\overline{\mathbf{Z}}$ \\
\hline
\end{tabular}


Table S15. Statistical data (in ppm) of calculated ${ }^{1} \mathrm{H}$ and ${ }^{13} \mathrm{C}$ chemical shifts (relative to the standard used). These include RMS error, mean error, mean absolute error, maximum error and standard deviation of absolute error.

\begin{tabular}{|c|c|c|c|c|c|}
\hline & & ${ }^{1} \mathrm{H}$ & & & \\
\hline & TMS & $\begin{array}{c}2,2,3,3- \\
\text { 2H4]-3- } \\
\text { trimethylsily } \\
\text { 1-propanoate }\end{array}$ & DSS & TMS & $\begin{array}{c}2,2,3,3- \\
2 \mathrm{H} 4]-3- \\
\text { trimethylsily } \\
\text { 1-propanoate }\end{array}$ \\
\hline RMS error & 0.52 & 1.01 & 0.91 & 11.3 & 11.2 \\
\hline mean error & 0.11 & 0.77 & 0.38 & 5.9 & 8.4 \\
\hline mean absolute error & 0.44 & 0.78 & 0.69 & 9.5 & 10.0 \\
\hline maximum error & 1.08 & 1.84 & -1.75 & 27.0 & 17.3 \\
\hline $\begin{array}{l}\text { standard deviation of absolute } \\
\text { error }\end{array}$ & 0.27 & 0.71 & 0.63 & 6.4 & 5.4 \\
\hline
\end{tabular}

Table S16. Relative free energies (in $\mathrm{kcal} / \mathrm{mol}$ ), calculated as described in the Part (ii) of "Molecular docking - Monomer" section of Methods part in the main text, of model system of apo hCN1 active site and its modifications (based on the hCN1 crystal structure), where

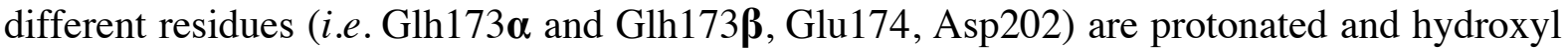
anion is bridging two $\mathrm{Zn}^{2+}$ ions. Relative free energies are calculated with respect to the model system, where water is bridging two $\mathrm{Zn}^{2+}$ ions and other residues are deprotonated.

\begin{tabular}{|c|c|c|c|c|}
\hline $\begin{array}{l}\text { Dielectric } \\
\text { constant }(\varepsilon)\end{array}$ & Glh173 $\boldsymbol{\alpha}$ & Glh173及 & Glu174 & Asp202 \\
\hline 1 & -13.0 & -0.9 & 38.9 & 37.4 \\
\hline 2 & -4.4 & 3.9 & 34.1 & 31.9 \\
\hline 4 & 0.6 & 6.4 & 30.5 & 28.3 \\
\hline 8 & 3.4 & 7.5 & 28.2 & 26.3 \\
\hline 16 & 4.8 & 8.1 & 26.9 & 25.2 \\
\hline 32 & 5.5 & 8.4 & 26.3 & 24.6 \\
\hline 64 & 5.9 & 8.5 & 25.9 & 24.3 \\
\hline 80 & 6.0 & 8.5 & 25.8 & 24.3 \\
\hline
\end{tabular}


Table S17. Relative free energies (in $\mathrm{kcal} / \mathrm{mol}$ ) of model system of apo hCN1 active site and its modifications (based on the hCN1 structure obtained after first step of docking), where

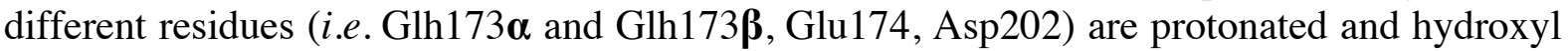
anion is bridging two $\mathrm{Zn}^{2+}$ ions. Relative free energies are calculated with respect to the model system, where water is bridging two $\mathrm{Zn}^{2+}$ ions and other residues are deprotonated.

\begin{tabular}{|c|c|c|c|c|}
\hline $\begin{array}{l}\text { Dielectric } \\
\text { constant }(\varepsilon)\end{array}$ & Glh173 $\boldsymbol{\alpha}$ & Glh173ß & Glu174 & Asp202 \\
\hline 1 & -29.2 & -32.3 & 9.8 & 4.2 \\
\hline 2 & -22.9 & -25.2 & 5.3 & 0.8 \\
\hline 4 & -19.1 & -20.6 & 2.7 & -1.1 \\
\hline 8 & -17.1 & -18.1 & 1.2 & -2.1 \\
\hline 16 & -16.0 & -16.7 & 0.4 & -2.6 \\
\hline 32 & -15.5 & -16.0 & 0.0 & -2.9 \\
\hline 64 & -15.2 & -15.7 & -0.2 & -3.0 \\
\hline 80 & -15.1 & -15.6 & -0.3 & -3.1 \\
\hline
\end{tabular}


Table S18. Hydrogen bond donors and acceptors inside the hCN1 active site from docking simulations. Asterisk indicates that the salt bridge is formed. HIE, HID and HIP indicate at which nitrogen atom the imidazole ring is protonated: HIE - protonated on Ne; HID -

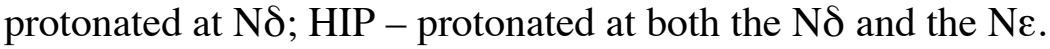

\begin{tabular}{|c|c|c|c|c|c|c|c|c|c|c|c|}
\hline \multirow{4}{*}{$\begin{array}{l}\text { Hydrogen } \\
\text { bond } \\
\text { donor }\end{array}$} & \multirow{4}{*}{$\begin{array}{l}\text { Hydrogen } \\
\text { bond } \\
\text { acceptor }\end{array}$} & \multicolumn{8}{|c|}{ Monomeric hCN1 } & \multirow{2}{*}{\multicolumn{2}{|c|}{$\begin{array}{c}\text { Dimeric hCN1 } \\
\mathrm{hCN} 1 \boldsymbol{\alpha}^{a}\end{array}$}} \\
\hline & & \multicolumn{6}{|c|}{ hCN1 $\alpha$} & \multicolumn{2}{|c|}{ hCN1/ } & & \\
\hline & & \multicolumn{3}{|c|}{ Lcar } & \multicolumn{3}{|c|}{ Hcar } & \multirow{2}{*}{$\begin{array}{l}\text { Lcar } \\
\text { (HIE) }\end{array}$} & \multirow{2}{*}{$\begin{array}{l}\text { Hcar } \\
\text { (HIE) }\end{array}$} & \multirow{2}{*}{$\begin{array}{c}\text { Lcar } \\
\text { (HIE) }\end{array}$} & \multirow{2}{*}{$\begin{array}{l}\text { Hcar } \\
\text { (HIE) }\end{array}$} \\
\hline & & HIE & HID & HIP & HIE & HID & HIP & & & & \\
\hline $\begin{array}{l}\text { Ligand } \\
\text { histidine } \\
\text { ring }\end{array}$ & $\begin{array}{l}\text { Glu173 } \\
\text { side chain }\end{array}$ & & $\checkmark$ & & & & & & & $\checkmark$ & \\
\hline $\begin{array}{l}\text { Ligand } \\
\text { histidine } \\
\text { ring }\end{array}$ & $\begin{array}{c}\text { Glu174 } \\
\text { backbone } \\
\text { oxygen }\end{array}$ & $\checkmark$ & & $\checkmark$ & $\boldsymbol{V}$ & & $\checkmark$ & & $v$ & & $\checkmark$ \\
\hline $\begin{array}{l}\text { Ligand } \\
\text { amino } \\
\text { group }\end{array}$ & $\begin{array}{c}\text { Asp202 } \\
\text { backbone } \\
\text { oxygen }\end{array}$ & & & & & & $v$ & & $\checkmark$ & & $\checkmark$ \\
\hline $\begin{array}{l}\text { Ligand } \\
\text { amino } \\
\text { group }\end{array}$ & $\begin{array}{c}\text { Asn203 } \\
\text { side chain }\end{array}$ & & & & $\checkmark$ & $v$ & & $\checkmark$ & $\checkmark$ & & \\
\hline $\begin{array}{l}\text { Arg350 } \\
\text { side chain }\end{array}$ & $\begin{array}{l}\text { Ligand } \\
\text { carboxyl } \\
\text { group }\end{array}$ & $\boldsymbol{V}^{*}$ & $\boldsymbol{V}^{*}$ & $\boldsymbol{V}^{*}$ & $\boldsymbol{V}^{*}$ & $\boldsymbol{V}^{*}$ & $\boldsymbol{V}^{*}$ & $\boldsymbol{V}^{*}$ & $\boldsymbol{V}^{*}$ & $\boldsymbol{V}^{*}$ & $\boldsymbol{V}^{*}$ \\
\hline $\begin{array}{c}\text { Ligand } \\
\text { peptide } \\
\text { NH group }\end{array}$ & $\begin{array}{c}\text { Thr424 } \\
\text { backbone } \\
\text { oxygen }\end{array}$ & $\checkmark$ & $\checkmark$ & $\checkmark$ & $\boldsymbol{V}$ & $\boldsymbol{V}$ & $\checkmark$ & $\checkmark$ & $\checkmark$ & $\checkmark$ & $\checkmark$ \\
\hline $\begin{array}{l}\text { Thr424 } \\
\text { peptide } \\
\text { NH group }\end{array}$ & $\begin{array}{l}\text { Ligand } \\
\text { carboxyl } \\
\text { group }\end{array}$ & $\checkmark$ & $\checkmark$ & $\checkmark$ & $\boldsymbol{v}$ & 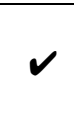 & $\checkmark$ & $v$ & $\checkmark$ & $\checkmark$ & $\checkmark$ \\
\hline $\begin{array}{l}\text { Hydroxide } \\
\text { anion }\end{array}$ & $\begin{array}{c}\text { Ligand } \\
\text { backbone } \\
\text { oxygen }\end{array}$ & $v$ & $\checkmark$ & $\checkmark$ & $\checkmark$ & $\checkmark$ & $\checkmark$ & $\checkmark$ & $\checkmark$ & & \\
\hline $\begin{array}{l}\text { His235 } \\
\text { imidazole } \\
\text { ring }\end{array}$ & $\begin{array}{l}\text { Ligand } \\
\text { peptide } \\
\text { oxygen }\end{array}$ & & & & & & & & & $\checkmark$ & $\checkmark$ \\
\hline $\begin{array}{l}\text { Total n } \\
\text { hydrog }\end{array}$ & $\begin{array}{l}\text { mber of } \\
n \text { bonds }\end{array}$ & 5 & 5 & 5 & 6 & 5 & 6 & 5 & 7 & 5 & 6 \\
\hline
\end{tabular}

${ }^{a}$ data is shown for LBP1 and HBP1. 
Table S19. Distances (in $\AA$ ) between two zinc ions and their coordinating residues from our 2-ps long QM/MM simulations of Lcar and Hcar complexes with monomeric and dimeric hCN1 $\alpha$ at $298 \mathrm{~K}$ and crystallographic data of apo-enzyme (PDB ID 3DLJ). Bond lengths from simulations are reported as average values.

\begin{tabular}{|c|c|c|c|c|c|}
\hline \multirow{2}{*}{ bond } & \multirow{2}{*}{$\begin{array}{l}\text { crystallographic } \\
\text { data }\end{array}$} & \multicolumn{2}{|c|}{ Monomeric hCN1 $\alpha$} & \multicolumn{2}{|c|}{ Dimeric hCN1 $\alpha$} \\
\hline & & Lcar $\bullet$ hCN1 $\boldsymbol{\alpha}$ & Hcar $\bullet$ hCN1 $\boldsymbol{\alpha}$ & Lcar $\bullet$ hCN1 $\boldsymbol{\alpha}$ & $\mathrm{Hcar} \bullet \mathrm{hCN} 1 \boldsymbol{\alpha}$ \\
\hline Zn1-His452 & 2.0 & $2.0 \pm 0.1$ & $2.0 \pm 0.1$ & $2.0 \pm 0.1$ & $2.0 \pm 0.1$ \\
\hline Zn1-Glu174 & 2.1 & $2.0 \pm 0.1$ & $2.0 \pm 0.1$ & $2.1 \pm 0.1$ & $2.0 \pm 0.1$ \\
\hline Zn1-Asp139 & 2.2 & $4.2 \pm 0.5$ & $4.1 \pm 0.2$ & $2.1 \pm 0.1$ & $2.1 \pm 0.1$ \\
\hline$\overline{\mathrm{Zn} 1-\mathrm{OH}^{-}}$ & 2.8 & $2.0 \pm 0.1$ & $2.0 \pm 0.1$ & $2.0 \pm 0.1$ & $2.1 \pm 0.1$ \\
\hline Zn2-Asp139 & 2.0 & $2.1 \pm 0.1$ & $2.1 \pm 0.1$ & $2.0 \pm 0.1$ & $2.0 \pm 0.1$ \\
\hline Zn2-Asp202 & 1.8 & $2.2 \pm 0.1$ & $2.1 \pm 0.1$ & $2.5 \pm 0.5$ & $2.1 \pm 0.1$ \\
\hline Zn2-His106 & 2.0 & $2.2 \pm 0.1$ & $2.1 \pm 0.1$ & $2.1 \pm 0.1$ & $2.1 \pm 0.1$ \\
\hline $\mathrm{Zn} 2-\mathrm{OH}^{-}$ & 2.8 & $2.2 \pm 0.1$ & $2.2 \pm 0.1$ & $2.1 \pm 0.1$ & $2.1 \pm 0.1$ \\
\hline $\begin{array}{l}\text { Zn1-ligand's } \\
\text { carbonyl } \\
\text { oxygen }\end{array}$ & l & $4.2 \pm 0.3$ & $4.0 \pm 0.4$ & $5.1 \pm 0.3$ & $4.5 \pm 0.3$ \\
\hline $\mathrm{Zn} 1-\mathrm{Zn} 2$ & 3.5 & $3.8 \pm 0.1$ & $3.5 \pm 0.1$ & $3.4 \pm 0.1$ & $3.3 \pm 0.1$ \\
\hline
\end{tabular}


Table S20. Distances (in $\AA$ ) between two zinc ions and their coordinating residues from our 1-ps long QM/MM simulations of Lcar complex with monomeric hCN1 $\alpha$ at $298 \mathrm{~K}$ and 303 $\mathrm{K}$, Lcar and Hcar complex with monomeric hCN1ß at $298 \mathrm{~K}$ and crystallographic data of apo-enzyme (PDB ID 3DLJ). Bond lengths from simulations are reported as average values.

\begin{tabular}{|c|c|c|c|c|c|}
\hline bond & $\begin{array}{l}\text { crystallographic } \\
\text { data }\end{array}$ & $\begin{array}{c}\text { Lcar } \bullet \text { hCN1 } \alpha \\
(\mathrm{T}=298 \mathrm{~K})\end{array}$ & $\begin{array}{c}\text { Lcar } \bullet \text { hCN1 } \alpha \\
(\mathrm{T}=303 \mathrm{~K})\end{array}$ & $\begin{array}{c}\text { Lcar } \bullet \text { hCN1及 } \\
(\mathrm{T}=298 \mathrm{~K})\end{array}$ & 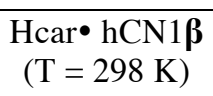 \\
\hline Zn1-His452 & 2.0 & $2.0 \pm 0.1$ & $2.0 \pm 0.1$ & $2.1 \pm 0.1$ & $2.0 \pm 0.1$ \\
\hline Zn1-Glu174 & 2.1 & $2.0 \pm 0.1$ & $2.0 \pm 0.1$ & $2.0 \pm 0.1$ & $2.1 \pm 0.1$ \\
\hline Zn1-Asp139 & 2.2 & $3.8 \pm 0.2$ & $3.8 \pm 0.2$ & $2.1 \pm 0.1$ & $3.7 \pm 0.3$ \\
\hline$\overline{\mathrm{Zn} 1-\mathrm{OH}^{-}}$ & 2.8 & $2.0 \pm 0.1$ & $2.0 \pm 0.1$ & $2.0 \pm 0.1$ & $2.0 \pm 0.1$ \\
\hline Zn2-Asp139 & 2.0 & $2.1 \pm 0.1$ & $2.1 \pm 0.1$ & $2.1 \pm 0.1$ & $2.0 \pm 0.1$ \\
\hline Zn2-Asp202 & 1.8 & $2.2 \pm 0.1$ & $2.2 \pm 0.1$ & $2.1 \pm 0.1$ & $2.2 \pm 0.1$ \\
\hline Zn2-His106 & 2.0 & $2.1 \pm 0.1$ & $2.1 \pm 0.1$ & $2.2 \pm 0.1$ & $2.2 \pm 0.1$ \\
\hline$\overline{\mathrm{Zn} 2-\mathrm{OH}^{-}}$ & 2.8 & $2.1 \pm 0.1$ & $2.1 \pm 0.1$ & $2.1 \pm 0.1$ & $2.1 \pm 0.1$ \\
\hline $\begin{array}{l}\text { Zn1-ligand's } \\
\text { carbonyl } \\
\text { oxygen }\end{array}$ & / & $4.1 \pm 0.2$ & $4.1 \pm 0.2$ & $5.5 \pm 0.4$ & $4.0 \pm 0.3$ \\
\hline Zn1-Zn2 & 3.5 & $3.8 \pm 0.1$ & $3.8 \pm 0.1$ & $3.3 \pm 0.1$ & $3.6 \pm 0.1$ \\
\hline
\end{tabular}


Table S21. Angles (in ${ }^{\circ}$ ) between two zinc ions and their coordinating residues from our 2-ps long QM/MM simulations of Lcar and Hcar complexes with monomeric and dimeric hCN1 $1 \alpha$ at $298 \mathrm{~K}$ and crystallographic data of apo-enzyme (PDB ID 3DLJ). Angle values from simulations are reported as average values.

\begin{tabular}{|c|c|c|c|c|c|}
\hline \multirow{2}{*}{ angle } & \multirow{2}{*}{$\begin{array}{c}\text { crystallographic } \\
\text { data }\end{array}$} & \multicolumn{2}{|c|}{ Monomeric hCN1 $\alpha$} & \multicolumn{2}{|c|}{ Dimeric hCN1 $\boldsymbol{\alpha}$} \\
\hline & & $\mathrm{Lcar} \bullet \mathrm{hCN} 1 \boldsymbol{\alpha}$ & Hcar $\bullet$ hCN1 $\boldsymbol{\alpha}$ & Lcar $\bullet$ hCN1 $1 \boldsymbol{\alpha}$ & Hcar $\bullet$ hCN $1 \alpha$ \\
\hline $\begin{array}{l}\text { His452-Zn1- } \\
\text { Glu174 }\end{array}$ & 115 & $122 \pm 6$ & $129 \pm 6$ & $119 \pm 7$ & $120 \pm 8$ \\
\hline $\begin{array}{l}\text { His452-Zn1- } \\
\text { Asp139 }\end{array}$ & 83 & $80 \pm 4$ & $66 \pm 5$ & $98 \pm 4$ & $97 \pm 5$ \\
\hline $\begin{array}{c}\text { His452-Zn1- } \\
\mathrm{OH}^{-}\end{array}$ & 115 & $124 \pm 9$ & $120 \pm 5$ & $121 \pm 8$ & $115 \pm 6$ \\
\hline $\begin{array}{l}\text { Glu174-Zn1- } \\
\text { Asp139 }\end{array}$ & 101 & $97 \pm 12$ & $95 \pm 11$ & $94 \pm 8$ & $101 \pm 6$ \\
\hline $\begin{array}{l}\text { Glu174-Zn1- } \\
\mathrm{OH}^{-}\end{array}$ & 126 & $109 \pm 7$ & $103 \pm 6$ & $112 \pm 6$ & $110 \pm 7$ \\
\hline $\begin{array}{c}\text { Asp139-Zn1- } \\
\mathrm{OH}^{-}\end{array}$ & 103 & $74 \pm 8$ & $85 \pm 6$ & $106 \pm 6$ & $111 \pm 7$ \\
\hline $\begin{array}{l}\text { His106-Zn2- } \\
\text { Asp139 }\end{array}$ & 106 & $91 \pm 4$ & $94 \pm 5$ & $114 \pm 9$ & $116 \pm 6$ \\
\hline $\begin{array}{l}\text { His106-Zn2- } \\
\text { Asp202 }\end{array}$ & 102 & $92 \pm 5$ & $101 \pm 5$ & $122 \pm 11$ & $114 \pm 9$ \\
\hline $\begin{array}{c}\text { His106-Zn2- } \\
\mathrm{OH}^{-}\end{array}$ & 128 & $100 \pm 7$ & $89 \pm 4$ & $97 \pm 4$ & $98 \pm 8$ \\
\hline $\begin{array}{l}\text { Asp139-Zn2- } \\
\text { Asp-202 }\end{array}$ & 127 & $139 \pm 12$ & $119 \pm 7$ & $111 \pm 9$ & $112 \pm 7$ \\
\hline $\begin{array}{c}\text { Asp139-Zn2- } \\
\mathrm{OH}^{-}\end{array}$ & 101 & $102 \pm 9$ & $141 \pm 9$ & $101 \pm 6$ & $95 \pm 7$ \\
\hline $\begin{array}{c}\text { Asp202-Zn2- } \\
\mathrm{OH}^{-}\end{array}$ & 96 & $117 \pm 8$ & $99 \pm 8$ & $105 \pm 12$ & $117 \pm 9$ \\
\hline
\end{tabular}


Table S22. Angles (in ${ }^{\circ}$ ) between two zinc ions and their coordinating residues from our 1-ps long QM/MM simulations of Lcar complex with monomeric hCN1 $\alpha$ at $298 \mathrm{~K}$ and $303 \mathrm{~K}$, Lcar and Hcar complex with monomeric form of hCN1 1 at $298 \mathrm{~K}$ and crystallographic data of apo-enzyme (PDB ID 3DLJ). Bond lengths from simulations are reported as average values.

\begin{tabular}{|c|c|c|c|c|c|}
\hline angle & $\begin{array}{c}\text { crystallographic } \\
\text { data }\end{array}$ & $\begin{array}{c}\mathrm{Lcar} \bullet \mathrm{hCN} 1 \boldsymbol{\alpha} \\
(\mathrm{T}=298 \mathrm{~K})\end{array}$ & $\begin{array}{c}\text { Lcar } \bullet \text { hCN1 } \boldsymbol{\alpha} \\
(\mathrm{T}=303 \mathrm{~K})\end{array}$ & 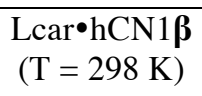 & $\begin{array}{c}\text { Hcar• hCN1 } \beta \\
(\mathrm{T}=298 \mathrm{~K})\end{array}$ \\
\hline $\begin{array}{l}\text { His452-Zn1- } \\
\text { Glu174 }\end{array}$ & 115 & $120 \pm 6$ & $119 \pm 7$ & $118 \pm 6$ & $111 \pm 9$ \\
\hline $\begin{array}{l}\text { His452-Zn1- } \\
\text { Asp139 }\end{array}$ & 83 & $78 \pm 4$ & $79 \pm 5$ & $100 \pm 6$ & $72 \pm 5$ \\
\hline $\begin{array}{l}\text { His452-Zn1- } \\
\mathrm{OH}^{-}\end{array}$ & 115 & $129 \pm 8$ & $129 \pm 9$ & $110 \pm 7$ & $135 \pm 15$ \\
\hline $\begin{array}{l}\text { Glu174-Zn1- } \\
\text { Asp139 }\end{array}$ & 101 & $107 \pm 7$ & $108 \pm 8$ & $108 \pm 6$ & $93 \pm 11$ \\
\hline $\begin{array}{l}\text { Glu174-Zn1- } \\
\mathrm{OH}^{-}\end{array}$ & 126 & $106 \pm 7$ & $107 \pm 7$ & $106 \pm 7$ & $104 \pm 10$ \\
\hline $\begin{array}{l}\text { Asp139-Zn1- } \\
\mathrm{OH}^{-}\end{array}$ & 103 & $68 \pm 6$ & $69 \pm 6$ & $114 \pm 4$ & $79 \pm 8$ \\
\hline $\begin{array}{l}\text { His106-Zn2- } \\
\text { Asp139 }\end{array}$ & 106 & $91 \pm 5$ & $91 \pm 5$ & $92 \pm 7$ & $97 \pm 5$ \\
\hline $\begin{array}{l}\text { His106-Zn2- } \\
\text { Asp202 }\end{array}$ & 102 & $93 \pm 5$ & $93 \pm 4$ & $117 \pm 4$ & $97 \pm 6$ \\
\hline $\begin{array}{l}\text { His106-Zn2- } \\
\mathrm{OH}^{-}\end{array}$ & 128 & $102 \pm 7$ & $102 \pm 8$ & $93 \pm 5$ & $94 \pm 9$ \\
\hline $\begin{array}{l}\text { Asp139-Zn2- } \\
\text { Asp-202 }\end{array}$ & 127 & $134 \pm 11$ & $136 \pm 10$ & $93 \pm 9$ & $122 \pm 7$ \\
\hline $\begin{array}{l}\text { Asp139-Zn2- } \\
\mathrm{OH}^{-}\end{array}$ & 101 & $105 \pm 7$ & $104 \pm 8$ & $99 \pm 7$ & $115 \pm 5$ \\
\hline $\begin{array}{l}\text { Asp202-Zn2- } \\
\mathrm{OH}^{-}\end{array}$ & 96 & $118 \pm 8$ & $118 \pm 7$ & $135 \pm 12$ & $118 \pm 10$ \\
\hline
\end{tabular}


Table S23. Probability of hydrogen bonds between the ligand and the hCN1 $\beta$ active site from 1-ps QM/MM simulations. ${ }^{\mathrm{WM}}$ indicates water mediated hydrogen bond.

\begin{tabular}{|c|c|c|c|}
\hline $\begin{array}{l}\text { Hydrogen bond } \\
\text { donor }\end{array}$ & $\begin{array}{l}\text { Hydrogen bond } \\
\text { acceptor }\end{array}$ & Lcar $\bullet$ hCN1及 & Hcar $\bullet$ hCN1 $\beta$ \\
\hline $\begin{array}{l}\text { Glh173 } \\
\text { protonated } \\
\text { carboxylic } \\
\text { group }\end{array}$ & $\begin{array}{l}\text { Ligand } \\
\text { backbone } \\
\text { oxygen }\end{array}$ & / & $100 \%$ \\
\hline $\begin{array}{l}\text { Ligand } \\
\text { histidine ring }\end{array}$ & $\begin{array}{c}\text { Glu174 } \\
\text { backbone } \\
\text { oxygen }\end{array}$ & $90 \%$ & $\begin{array}{c}43 \% \\
79 \% \text { WM }\end{array}$ \\
\hline $\begin{array}{l}\text { Ligand amino } \\
\text { group }\end{array}$ & $\begin{array}{c}\text { Asp202 } \\
\text { backbone } \\
\text { oxygen }\end{array}$ & $91 \%$ & $\begin{array}{c}27 \% \\
68 \% \text { WM }\end{array}$ \\
\hline $\begin{array}{l}\text { Ligand amino } \\
\text { group }\end{array}$ & $\begin{array}{c}\text { Asp202 } \\
\text { carboxylic } \\
\text { group }\end{array}$ & / & I \\
\hline $\begin{array}{l}\text { Ligand amino } \\
\text { group }\end{array}$ & $\begin{array}{l}\text { Asn } 203 \text { side } \\
\text { chain }\end{array}$ & I & $8 \%$ \\
\hline $\begin{array}{l}\text { Arg350 side } \\
\text { chain* }\end{array}$ & $\begin{array}{l}\text { Ligands } \\
\text { carboxylic } \\
\text { group }\end{array}$ & $\begin{array}{c}\text { a) } / \\
\text { b) } 87 \% \\
\text { c) } 55 \% \\
\text { d) } 6 \%\end{array}$ & $\begin{array}{l}\text { a) } 14 \% \\
\text { b) } 28 \% \\
\text { c) } 32 \% \\
\text { d) } 12 \%\end{array}$ \\
\hline $\begin{array}{l}\text { Ligand amino } \\
\text { group }\end{array}$ & $\begin{array}{l}\text { Asp421 } \\
\text { carboxylic } \\
\text { group }\end{array}$ & / & $97 \%$ \\
\hline $\begin{array}{l}\text { Ligand amino } \\
\text { group }\end{array}$ & $\begin{array}{c}\text { Ser423 } \\
\text { hydroxyl group }\end{array}$ & / & $59 \%$ \\
\hline $\begin{array}{l}\text { Ligand peptide } \\
\text { NH group }\end{array}$ & $\begin{array}{c}\text { Thr424 } \\
\text { backbone } \\
\text { oxygen }\end{array}$ & $46 \%$ & $41 \%$ \\
\hline $\begin{array}{l}\text { Thr424 peptide } \\
\text { NH group }\end{array}$ & $\begin{array}{l}\text { Ligand } \\
\text { carboxylic } \\
\text { group }\end{array}$ & $29 \%$ & I \\
\hline
\end{tabular}

* between Arg350 and carboxylic group are 4 possible hydrogen bonds (marked with, a), b,)

c) and d), see also Fig. S8); also salt bridge is formed here. 
Table S24. Relative free energies (in $\mathrm{kcal} / \mathrm{mol}$ ) of hCN1 $\boldsymbol{\alpha}$ and $\mathrm{hCN} 1 \boldsymbol{\beta}$ of holo hCN1 active site (with Lcar and Hcar present inside the active site). Relative free energies were calculated with respect to the model system, where water is bridging two $\mathrm{Zn}^{2+}$ ions and Glu173 is deprotonated. The position of the hydrogen atoms was optimized. Single-point calculations were performed on the optimized structures. Values for the system with Hcar present inside the active site are in parentheses.

\begin{tabular}{|c|c|c|}
\hline $\begin{array}{c}\text { Dielectric } \\
\text { constant }(\varepsilon)\end{array}$ & 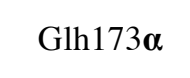 & Glh173及 \\
\hline 1 & $-24.8(-25.2)$ & $-4.3(-16.6)$ \\
\hline 2 & $-18.9(-19.3)$ & $-1.6(-8.0)$ \\
\hline 4 & $-15.3(-16.0)$ & $-0.1(-3.2)$ \\
\hline 8 & $-13.4(-13.8)$ & $0.7(-0.2)$ \\
\hline 16 & $-12.4(-12.8)$ & $1.1(1.0)$ \\
\hline 32 & $-11.8(-12.6)$ & $1.3(1.7)$ \\
\hline 64 & $-11.6(-12.1)$ & $1.4(2.3)$ \\
\hline 80 & $-11.5(-12.0)$ & $1.4(2.1)$ \\
\hline
\end{tabular}

Table S25. The average values of olvent accessible surface of monomeric and dimeric (monomer I) hCN1 from classical MD simulations.

\begin{tabular}{|l|c|c|}
\hline \multicolumn{1}{|c|}{ Ligand } & Monomeric hCN1 & Dimeric hCN1 \\
\hline Lcar & $251 \pm 3 \mathrm{~nm}^{2}$ & $253.3 \pm 3 \mathrm{~nm}^{2}$ \\
\hline Hcar & $249 \pm 3 \mathrm{~nm}^{2}$ & $250 \pm 2 \mathrm{~nm}^{2}$ \\
\hline
\end{tabular}




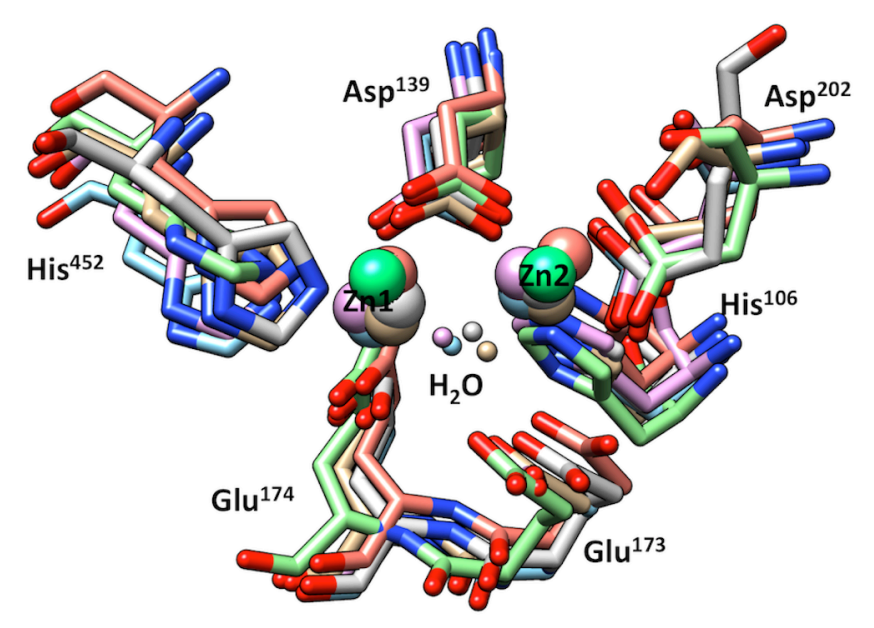

Figure S1. Superimposition of hCN1 active site (colored in tan) with other available crystal structures of metallopeptidases with the same active site. These are: the aminopeptidase from Aeromonas proteolytica (colored in blue; PDB ID 1AMP) ${ }^{19}$, the aminopeptidase from Streptomyces griseus (colored in purple; PDB ID 1CP7) ${ }^{20}$, the putative Xaa-His dipeptidase from Haemophilus somnus (colored in green; PDB ID 2QYV; without presence of crystal water molecules; article has not been yet published), the aminopetidase PepV from Lactobacillus delbrueckii (colored in red; PDB ID 1LFW; crystal structure has ligand inside active site and therefore bridging water molecule is not present $)^{21}$ and carboxypeptidase G2 from Pseudomonas species (colored in grey; PDB ID 1CG2). ${ }^{22}$ Zinc ions are represented as big spheres and bridging water molecules as small sphere, both in the same color as the corresponding enzyme. Numbering of the residues corresponds to the hCN1.

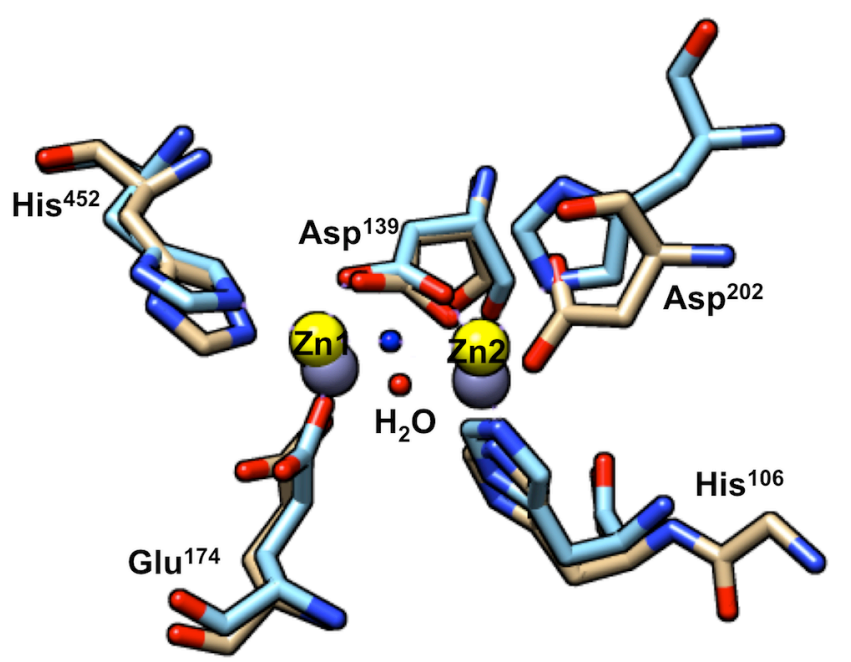

Figure S2. Superposition of hCN1 and yeast $\beta$-alanine-synthase active sites. ${ }^{23}$ hCN1's residues are shown in brown and labeled. The $\mathrm{Zn}^{2+}$ ions and water molecule are represented as violet and red spheres, respectively. y $\beta A S$ 's residues are colored in light blue; the $\mathrm{Zn}^{2+}$ ions and water molecule are shown as yellow and dark blue balls respectively. Residues are numbered as in hCN1. 

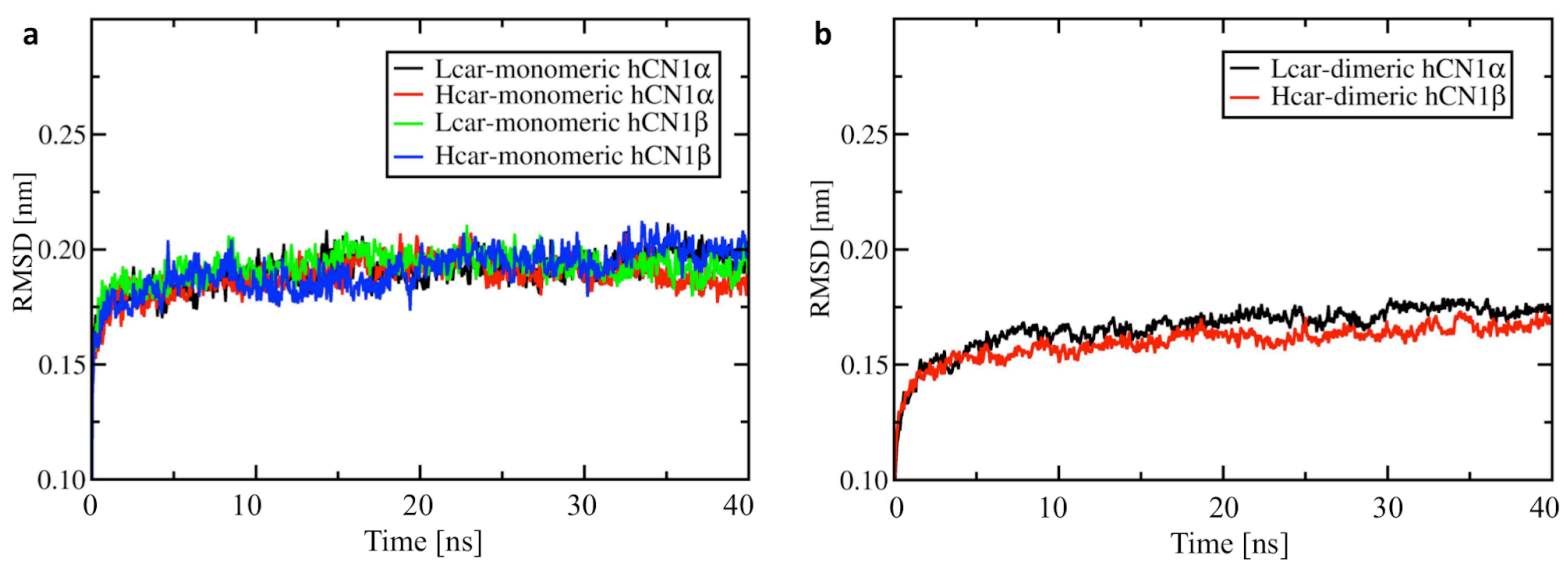

Figure S3. RMSD of hCN1 relative to the structure of the enzyme used in the second step of molecular docking during $40 \mathrm{~ns}$ classical MD. a) monomeric hCN1 complexes (Lcar $\bullet$ hCN1 $\boldsymbol{\alpha}$ in black, Lcar $\bullet$ hCN1 $\beta$ in green, Hcar $\bullet$ hCN1 $\alpha$ in red and Hcar $\bullet$ hCN1 $\beta$ in blue). b) dimeric hCN1 complexes (Lcar $\bullet$ hCN1 $\boldsymbol{\alpha}$ in black and Hcar $\bullet$ hCN1 $\boldsymbol{\alpha}$ in red).
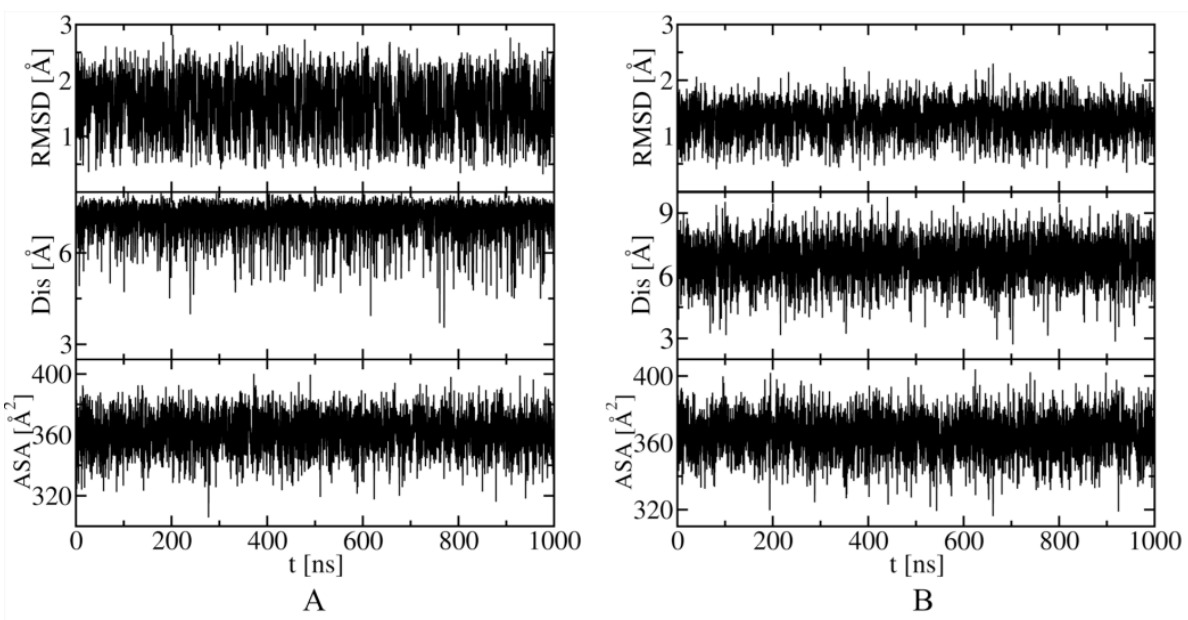

Figure S4. (a) RMSD, (b) distance between terminal $\mathrm{NH}_{3}{ }^{+}$and $\mathrm{COO}^{-}$, and (c) accessible surface area plotted as a function of simulated time for $\mathbf{A}$ and $\mathbf{B}$ Lcar tautomers in aqueous solution. The plots refer to only one of the two MD simulations reported here. 


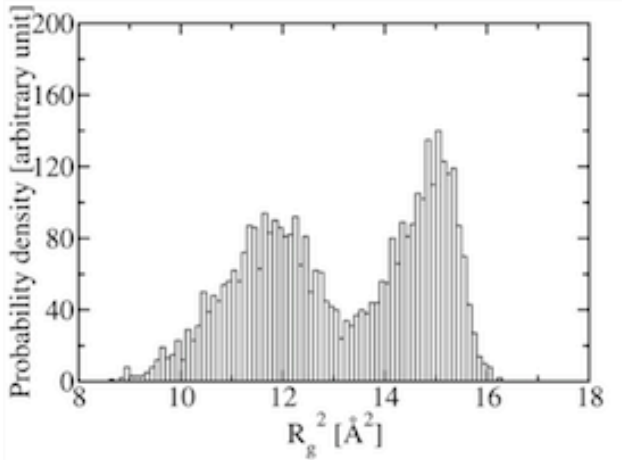

A

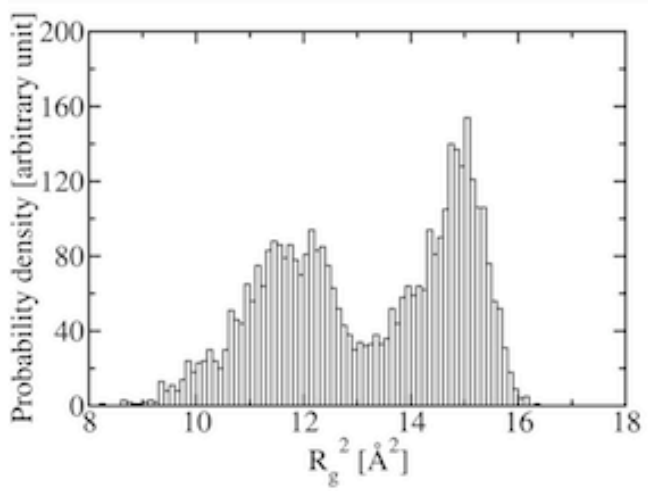

A

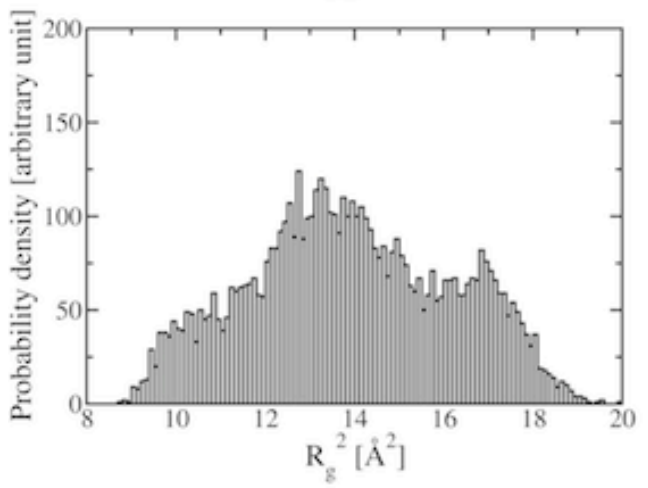

C

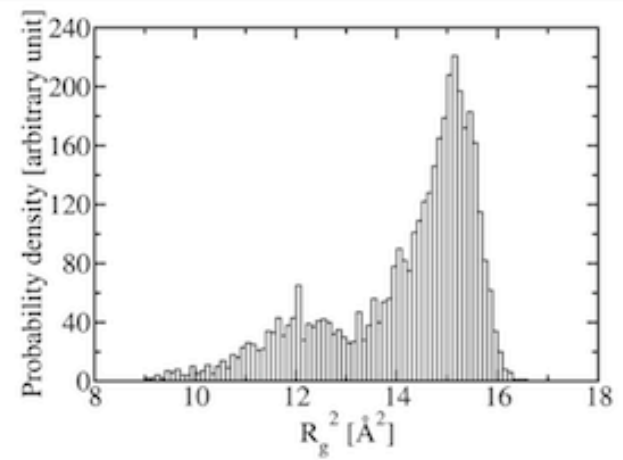

B

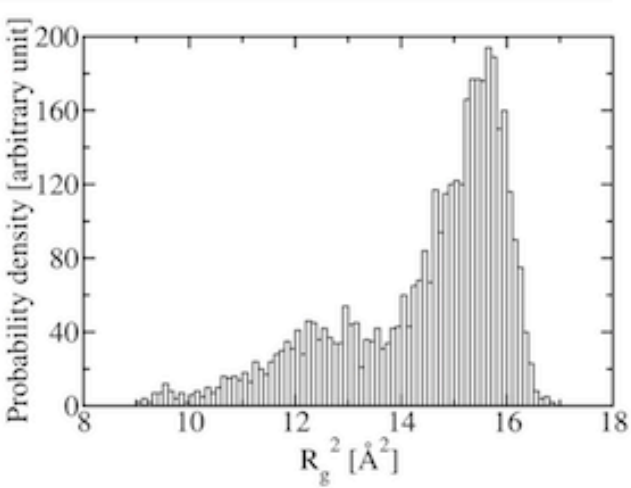

$\mathrm{B}$

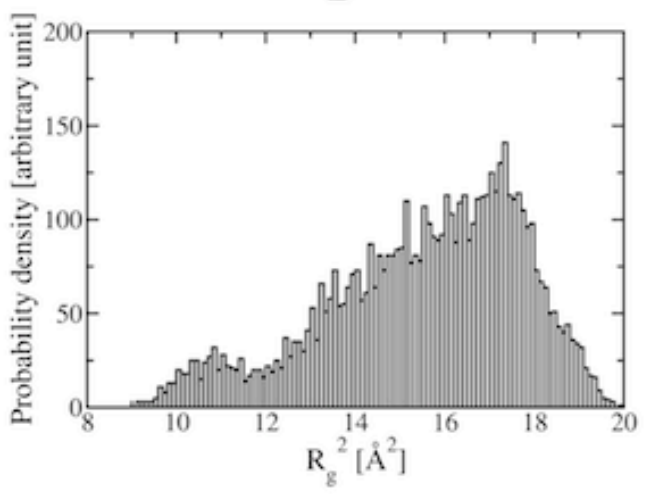

D

Figure S5. Probability distribution of $\mathbf{R}_{\mathrm{g}}{ }^{2}$ in $\mathbf{A}$ and $\mathbf{B}$ in aqueous solution (upper line) and comparison with independent simulations (middle line). Last line reports the probability distribution of $\mathbf{R}_{\mathrm{g}}{ }^{2}$ for $\mathbf{C}$ and $\mathbf{D}$. 


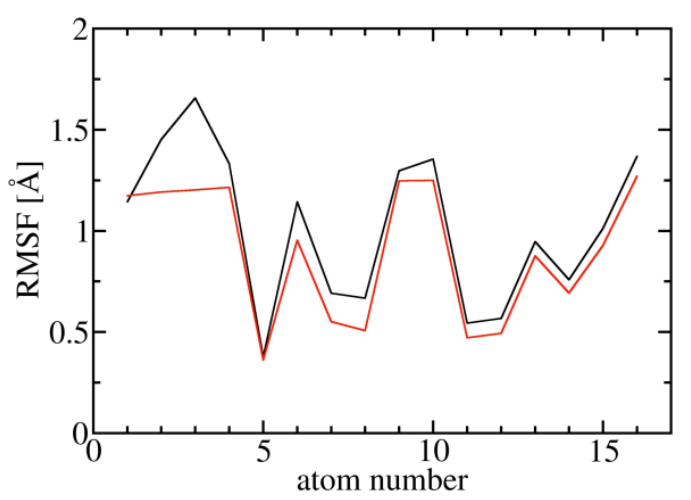

Figure S6. RMSF values of pairs of equivalent atoms (data shown only for $\mathrm{C}, \mathrm{N}$ and $\mathrm{O}$ atoms) in $\mathbf{A}$ (black line) and $\mathbf{B}$ (red line) tautomers of Lcar during one of the two MD simulations reported here. 
(a)
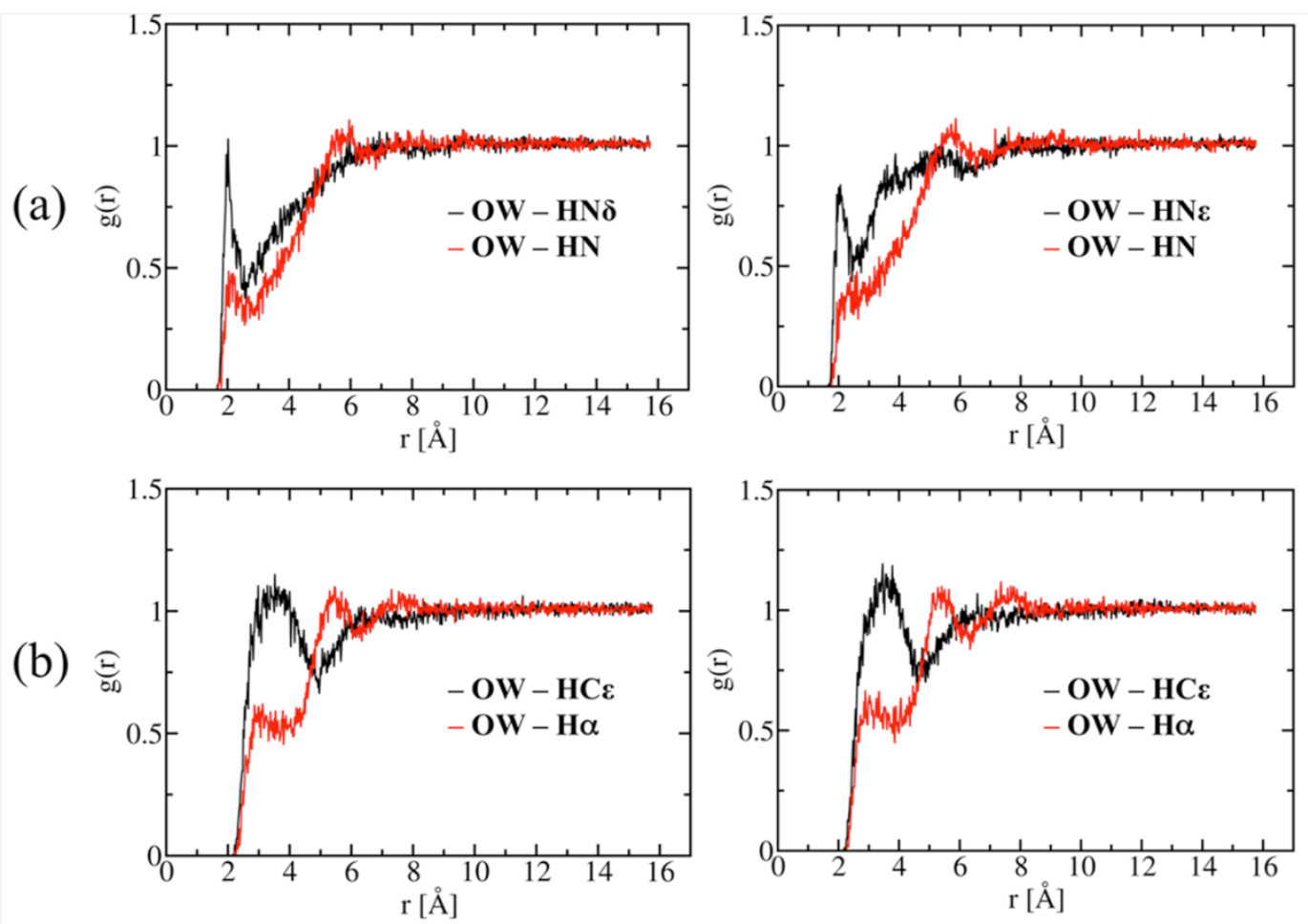

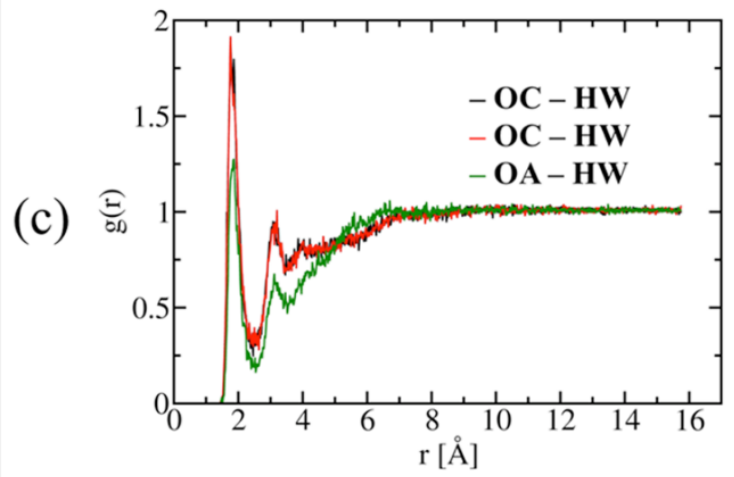

A

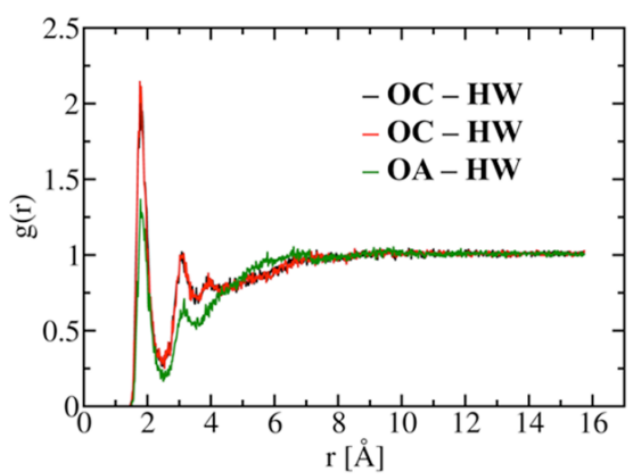

$\mathrm{B}$

Figure S7. Radial distribution functions $g(r)$ of $\mathbf{A}$ and $\mathbf{B}$ Lcar tautomers in water. (a) $g(r)$ between the N-hydrogen atom in imidazole ring ( $\mathrm{HN} \delta$ and $\mathrm{HN} \varepsilon$ ) of Lcar and oxygen atom in water (OW) (black line); $\mathrm{g}(\mathrm{r})$ of amide hydrogen (HN) of Lcar and oxygen atom in water (OW) (red line). (b) $\mathrm{g}(\mathrm{r})$ of the C-hydrogen atom in imidazole ring ( $\mathrm{HC} \varepsilon$ ) of Lcar and oxygen atom of water (OW) (black line); $\mathrm{g}(\mathrm{r})$ of $\mathrm{C} \alpha$-hydrogen of Lcar and oxygen atom in water (OW) (red line). (c) $\mathrm{g}(\mathrm{r})$ of both carboxylic oxygen atoms (OC) of Lcar and hydrogen atom in water (HW) (black and red lines); g(r) of amide oxygen atom (OA) of Lcar and hydrogen atom in water (HW) (green line). For the name of the atoms see Chart 1 in the Main Text. 

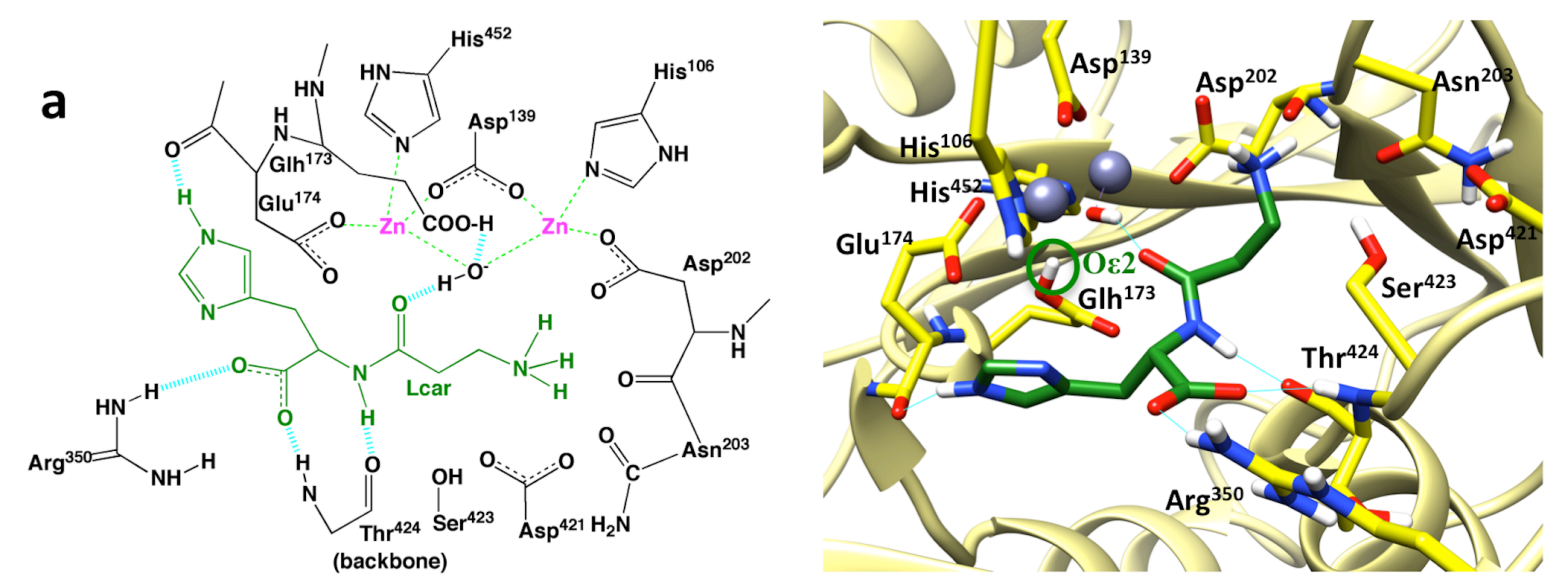

b

b
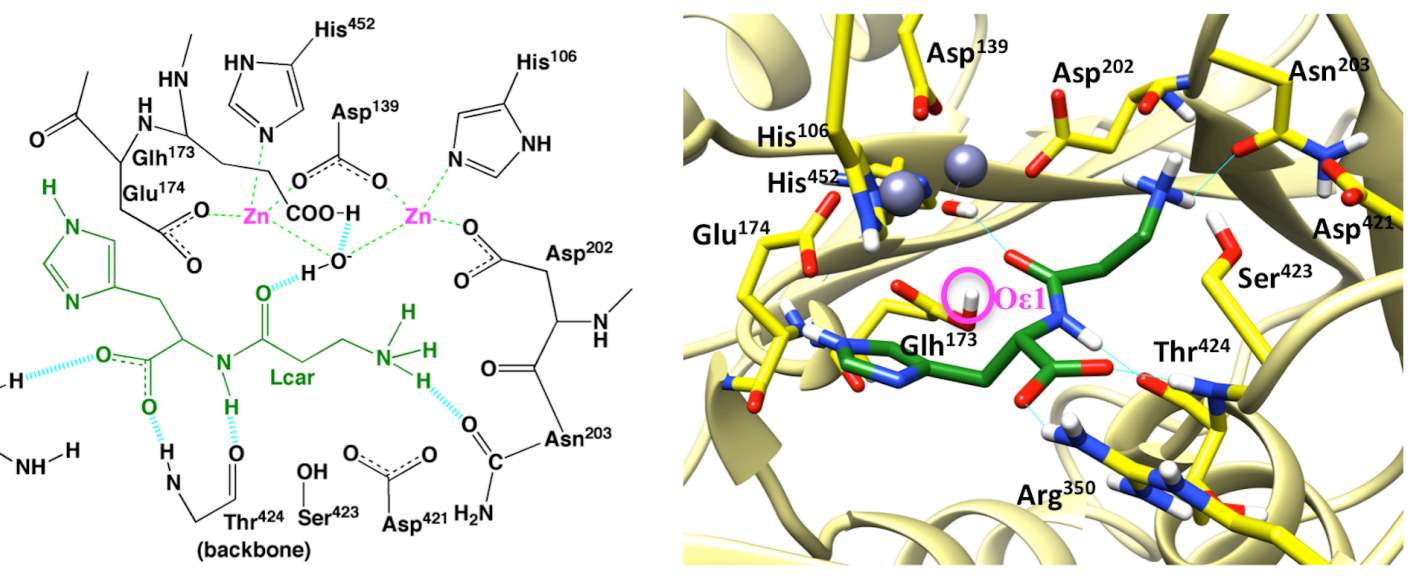

C
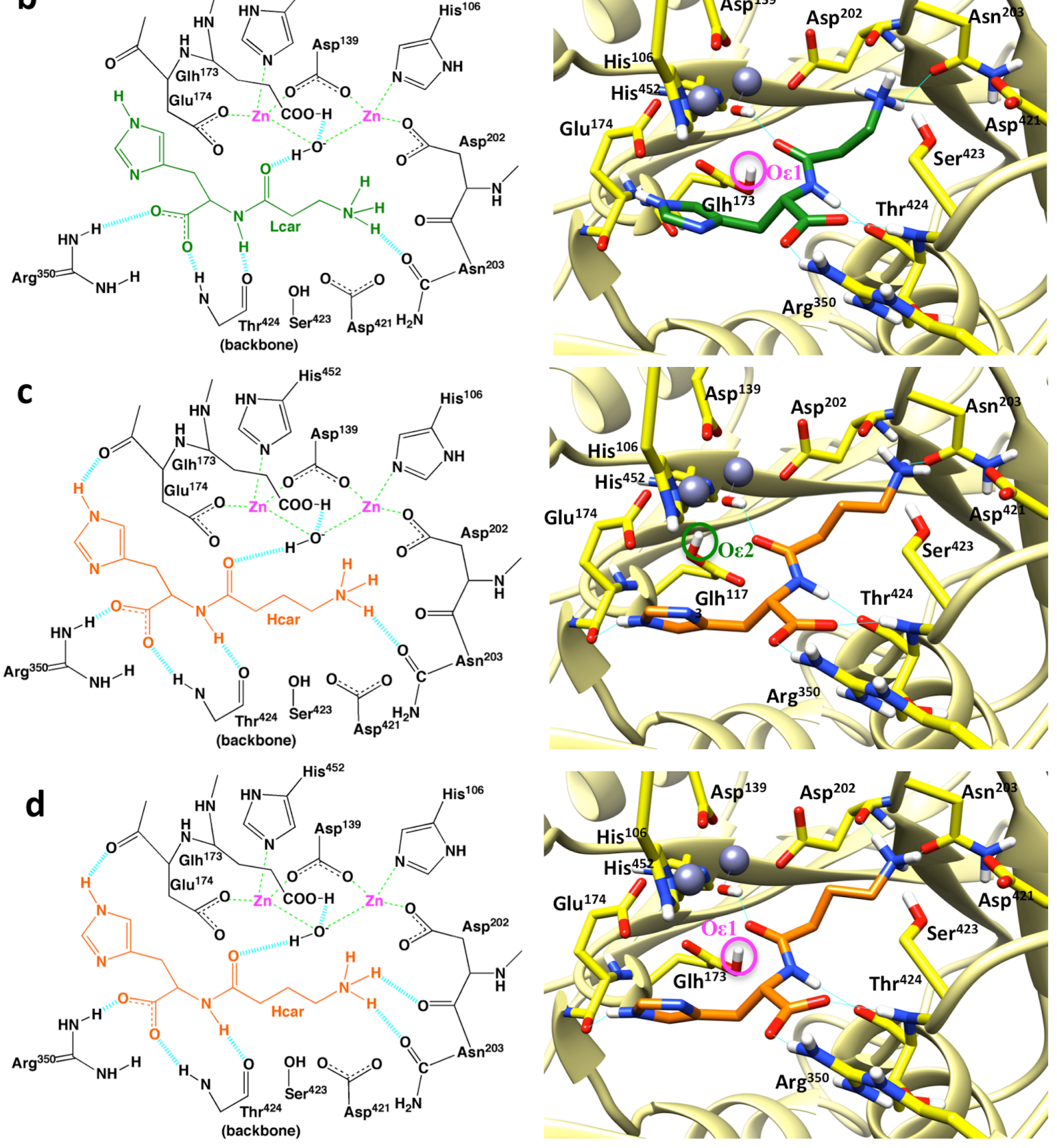
Figure S8. Highest populated clusters of monomeric hCN1 complexes (Lcar $\bullet \mathrm{hCN} 1 \boldsymbol{\alpha}(\mathrm{A})$, Lcar $\bullet$ hCN1 $\beta$ (B), Hcar $\bullet$ hN1 $\alpha$ (C) and Hcar $\bullet$ CN1 $\beta$ (D)) obtained by docking simulations

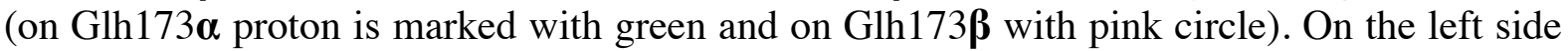
of the figure are schematic 2D representations of the complexes, while on the right side there are matching 3D figures. Lcar is shown in green and Hcar in orange. Hydrogen bonds are shown as dashed turquoise lines in $2 \mathrm{D}$ schemes and as full turquoise lines in the $3 \mathrm{D}$ representations. Coordination bonds between $\mathrm{Zn}^{2+}$ ions and hCN1 active site residues are shown on the $2 \mathrm{D}$ representations as dashed green lines. $\mathrm{Zn}^{2+}$ ions are shown as violet spheres in the $3 \mathrm{D}$ representation.
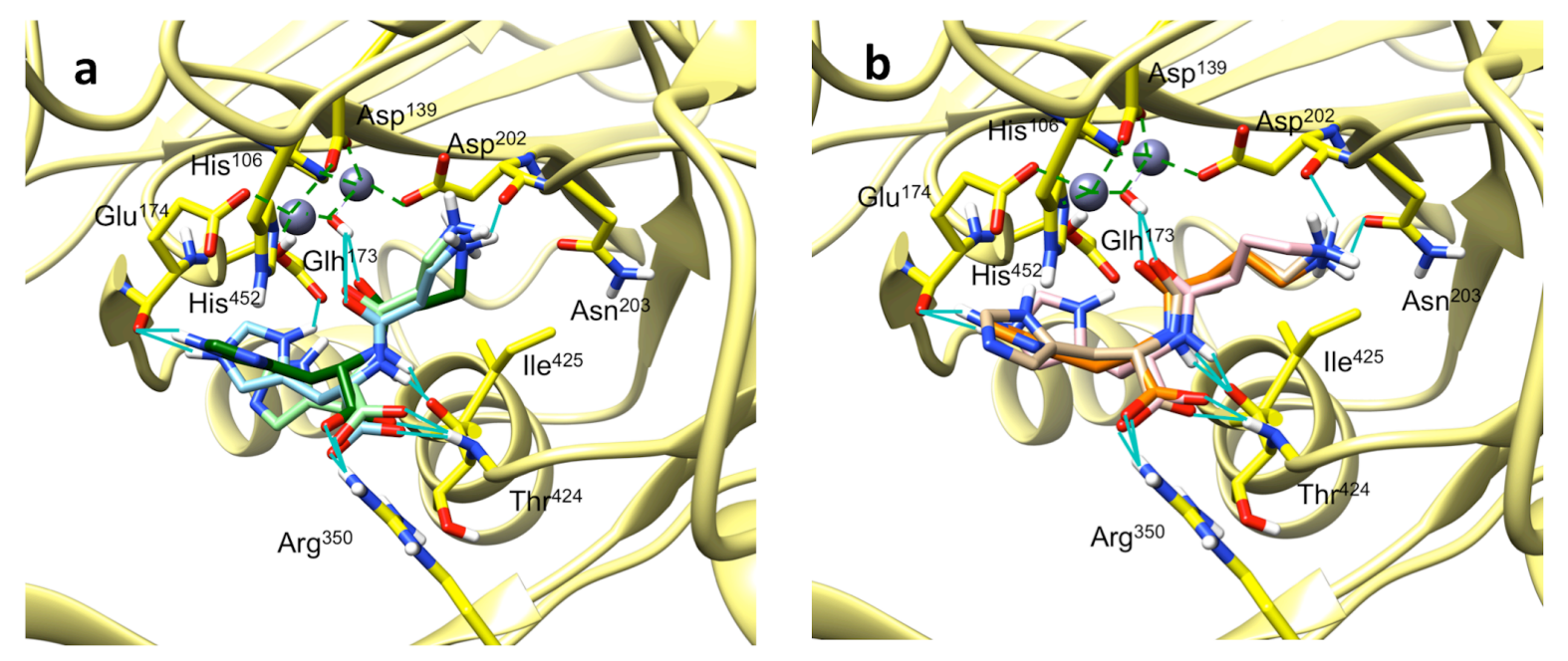

Figure S9. Highest populated clusters of monomeric hCN1 $\alpha$ complexes obtained by docking simulations. a) binding poses of Lcar in all three protonation states (HIE is shown in dark green, HID in light green and HIP in light blue); b) binding poses of Hcar in all three protonation states (HIE in shown in orange, HID in tan and HIP in light pink). Hydrogen bonds are shown as full turquoise lines. The coordination bonds between $\mathrm{Zn}^{2+}$ ions and hCN1 active site residues are shown as dashed green lines. The $\mathrm{Zn}^{2+}$ ions are shown as violet spheres. 

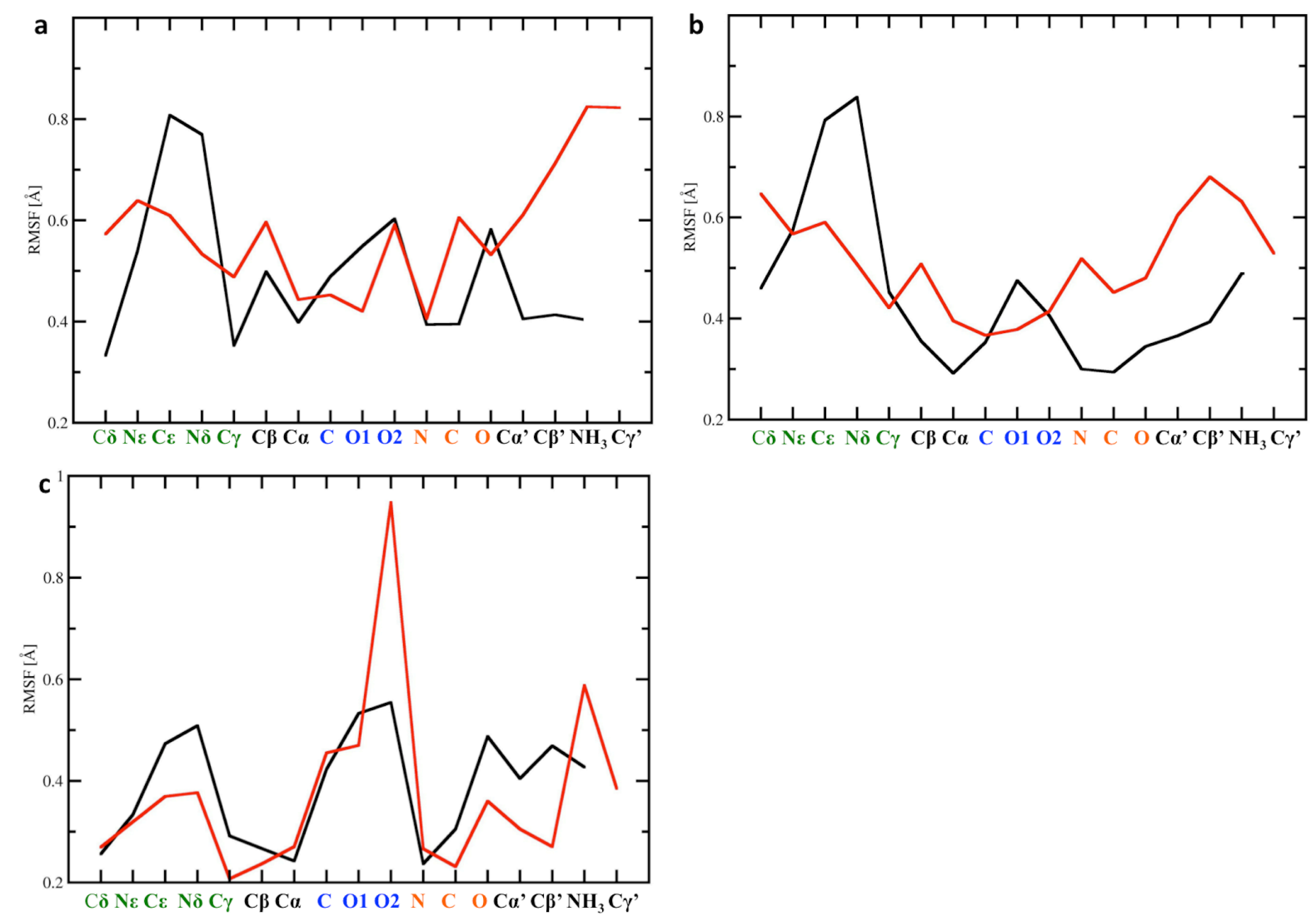

Figure S10. RMSF values for heavy atoms of both ligands inside a) monomeric hCN1 $\alpha$ from 2 ps long QM/MM simulations; b) dimeric hCN1 $\alpha$ from 2 ps long QM/MM simulations and c) monomeric hCN1/ from 1 ps long QM/MM simulations. Lcar's RMSF is shown in black and Hcar's in red. Naming of the atoms is the same as in Chart 1. Green colored atom names represent atoms of histidine ring, blue ones represent carboxylic group and orange one amide bond. 


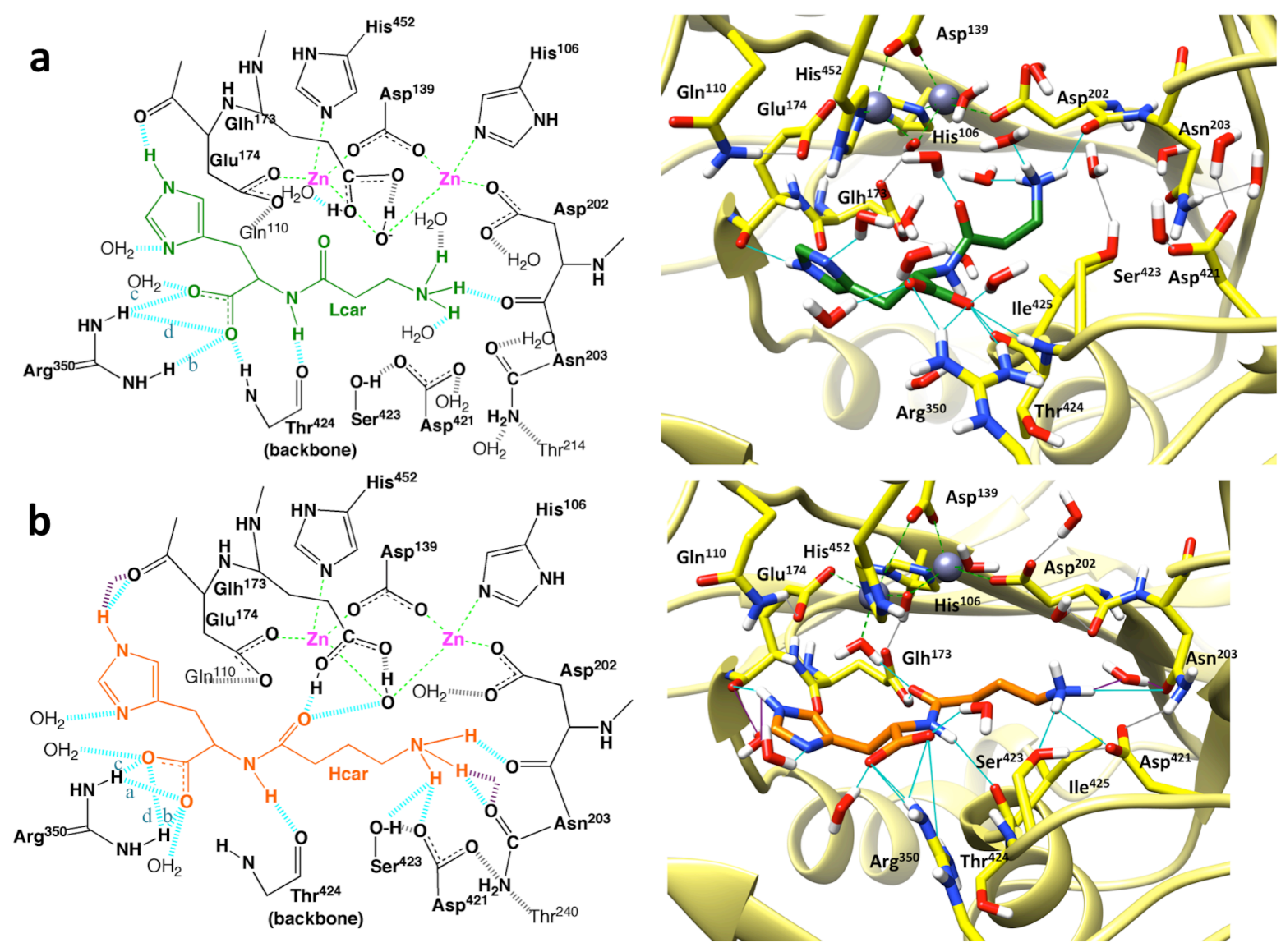

Figure S11. Structures of Lcar $\bullet$ monomeric hCN1及 (a) and Hcar $\bullet$ monomeric hCN1 $\beta$ (b) Michaelis complexes obtained by one $p s$-long QM/MM simulations. On the left side of the figure are schematic 2D representations of the Michaelis complexes, while on the right side there are matching 3D figures (non-polar hydrogen atoms are not shown due to clarity). Lcar is shown in green and Hcar in orange. Hydrogen bonds between ligand and environment are shown as dashed turquoise lines in 2D schemes and as full turquoise lines in the 3D representations (for occupancy of hydrogen bonds between ligands and hCN1 see Tab. S20; hydrogen bond naming ( $a, b, c$ and $d$ ) between ligand's carboxylic group and side chain of Arg376 correspond to the one in Tab. S20), while those between hCN1 residues and water molecules are colored in grey. Water-mediated hydrogen bonds are shown as dashed purple lines in 2D schemes (due to the clarity, water molecules are omitted in these schemes) and as full purple lines in the 3D figures. Coordination bonds between $\mathrm{Zn}^{2+}$ ions and hCN1 active site residues are shown as dashed green lines on all figures. $\mathrm{Zn}^{2+}$ ions are shown as violet spheres in the 3D representation. 

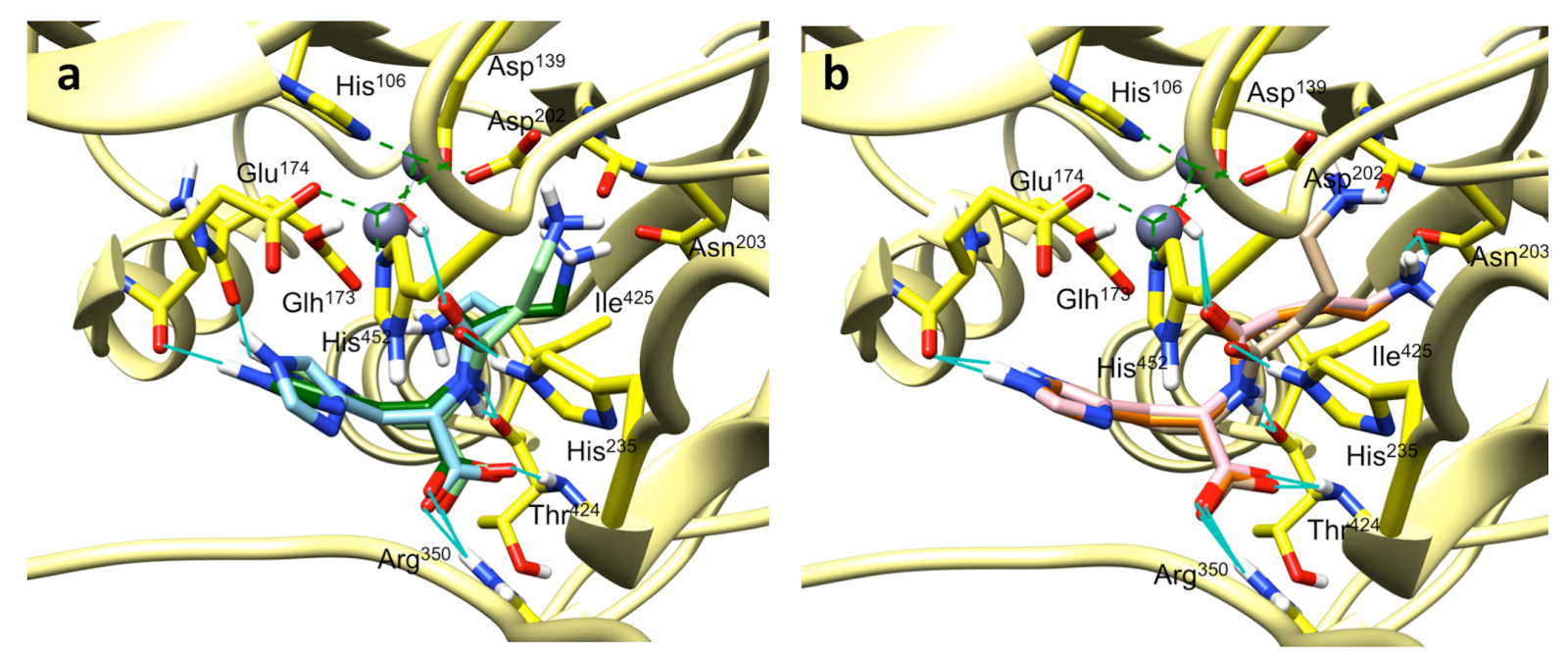

Figure S12. Dimeric hCN1 complexes in comparison with monomeric complexes obtained by docking simulations. a) binding poses of Lcar in hCN1 (Lcar $\bullet$ monomeric hCN1 $\boldsymbol{\alpha}$ is shown in dark green, LBP1 and LBP2 of Lcar $\bullet$ dimeric hCN1 $\boldsymbol{\alpha}$ are shown in light green and light blue, respectively); b) binding poses of Hcar in in hCN1 (Hcar $\bullet$ monomeric hCN1 $\alpha$ is shown in orange, HBP1 and HBP2 of Hcar ${ }^{-d i m e r i c ~ h C N 1} \boldsymbol{\alpha}$ are shown in tan and light pink, respectively). Hydrogen bonds are shown as full turquoise lines. Coordination bonds between $\mathrm{Zn}^{2+}$ ions and hCN1 active site residues are shown as dashed green lines. $\mathrm{Zn}^{2+}$ ions are shown as violet spheres. 


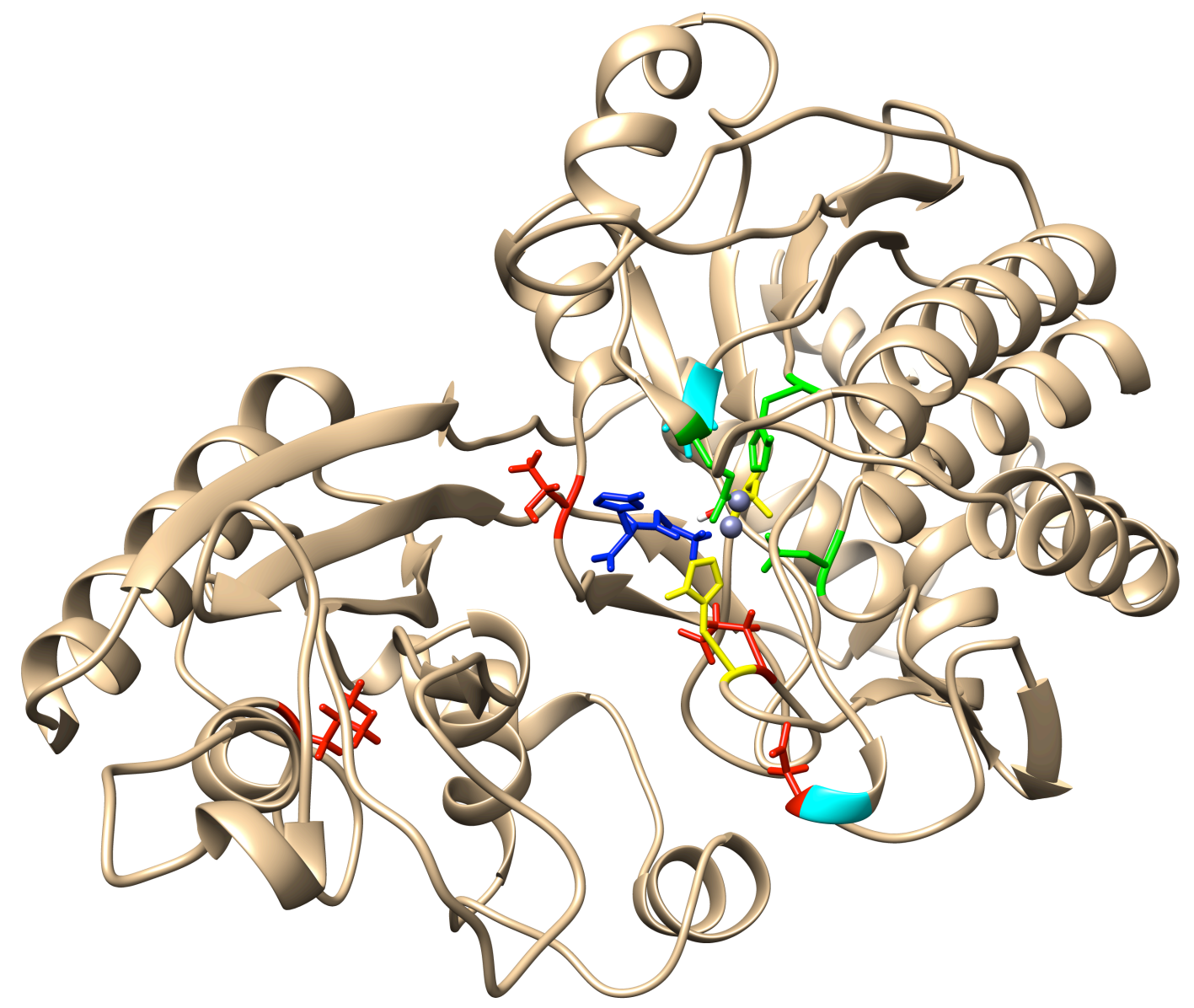

Figure S13. 1B representative of Lcar (colored in blue) docked in the monomeric hCN1 enzymatic cavity. $\mathrm{Zn}^{2+}$ ions are colored in pink. Residues coordinating $\mathrm{Zn}^{2+}$ according only to ref. $^{24}$ are colored in cyan and the ones according only to our model are colored in yellow. Common residues in the two models coordinating $\mathrm{Zn}^{2+}$ are colored in green. Residues coordinating Lcar according to ref. ${ }^{24}$ are colored in red. 


\section{Supporting References.}

1. Hess, B. (2002) Convergence of sampling in protein simulations, Physical Review E $65,031910$.

2. Cheeseman, J. R., Trucks, G. W., Keith, T. A., and Frisch, M. J. (1996) A comparison of models for calculating nuclear magnetic resonance shielding tensors, Journal of Chemical Physics 104, 5497-5509.

3. Frisch, M. J., Trucks, G. W., Schlegel, H. B., Scuseria, G. E., Robb, M. A., Cheeseman, J. R., Scalmani, G., Barone, V., Mennucci, B., Petersson, G. A., Nakatsuji, H., Caricato, M., Li, X., Hratchian, H. P., Izmaylov, A. F., Bloino, J., Zheng, G., Sonnenberg, J. L., Hada, M., Ehara, M., Toyota, K., Fukuda, R., Hasegawa, J., Ishida, M., Nakajima, T., Honda, Y., Kitao, O., Nakai, H., Vreven, T., Montgomery, J. A., Peralta, J. E., Ogliaro, F., Bearpark, M., Heyd, J. J., Brothers, E., Kudin, K. N., Staroverov, V. N., Kobayashi, R., Normand, J., Raghavachari, K., Rendell, A., Burant, J. C., Iyengar, S. S., Tomasi, J., Cossi, M., Rega, N., Millam, J. M., Klene, M., Knox, J. E., Cross, J. B., Bakken, V., Adamo, C., Jaramillo, J., Gomperts, R., Stratmann, R. E., Yazyev, O., Austin, A. J., Cammi, R., Pomelli, C., Ochterski, J. W., Martin, R. L., Morokuma, K., Zakrzewski, V. G., Voth, G. A., Salvador, P., Dannenberg, J. J., Dapprich, S., Daniels, A. D., Farkas, Foresman, J. B., Ortiz, J. V., Cioslowski, J., and Fox, D. J. (2009) Gaussian 09, revision A.02, Wallingford CT.

4. Zhang, R., Wang, D., and Wu, W. (2013) Conformations of carnosine in aqueous solutions by all-atom molecular dynamics simulations and 2D-NOSEY spectrum, Chinese Journal of Chemical Physics 26, 67-72.

5. Matsukura, T., Takahashi, T., Nishimura, Y., Ohtani, T., Sawada, M., and Shibata, K. (1990) Characterization of crystalline L-carnosine Zn(II) complex (Z-103), a novel anti-gastric ulcer agent: Tautomeric change of imidazole moiety upon complexation, Chemical and Pharmaceutical Bulletin 38, 3140-3146.

6. Diez, R. P., and Baran, E. J. (2003) A density functional study of some physical

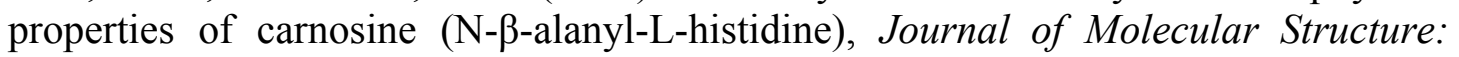
THEOCHEM 621, 245-251.

7. Verdonk, M. L., Cole, J. C., Hartshorn, M. J., Murray, C. W., and Taylor, R. D. (2003) Improved protein-ligand docking using GOLD, Proteins: Structure, Function, and Bioinformatics 52, 609-623.

8. Morris, G. M., Huey, R., Lindstrom, W., Sanner, M. F., Belew, R. K., Goodsell, D. S., and Olson, A. J. (2009) AutoDock4 and AutoDockTools4: Automated docking with selective receptor flexibility, Journal of Computational Chemistry 30, 2785-2791.

9. Santos-Martins, D., Forli, S., Ramos, M. J., and Olson, A. J. (2014) AutoDock4Zn: An improved AutoDock force field for small-molecule docking to zinc metalloproteins, Journal of Chemical Information and Modeling 54, 2371-2379.

10. Holz, R. C., Bzymek, K. P., and Swierczek, S. I. (2003) Co-catalytic metallopeptidases as pharmaceutical targets, Current Opinion in Chemical Biology 7, 197-206.

11. Navrátil, V., Klusák, V., and Rulíšek, L. (2013) Theoretical aspects of hydrolysis of peptide bonds by zinc metalloenzymes, Chemistry- A European Journal 19, 1663416645.

12. Teufel, M., Saudek, V., Ledig, J.-P., Bernhardt, A., Boularand, S., Carreau, A., Cairns, N. J., Carter, C., Cowley, D. J., Duverger, D., Ganzhorn, A. J., Guenet, C., Heintzelmann, B., Laucher, V., Sauvage, C., and Smirnova, T. (2003) Sequence 
identification and characterization of human carnosinase and a closely related nonspecific dipeptidase, Journal of Biological Chemistry 278, 6521-6531.

13. Pegova, A., Abe, H., and Boldyrev, A. (2000) Hydrolysis of carnosine and related compounds by mammalian carnosinases, Comparative Biochemistry and Physiology B: Biochemistry and Molecular Biology 127, 443-446.

14. Wang, J., Wolf, R. M., Caldwell, J. W., Kollman, P. A., and Case, D. A. (2004) Development and testing of a general amber force field, Journal of Computational Chemistry 25, 1157-1174.

15. Friedrich, J. O., and Wasylishen, R. E. (1986) A $1 \mathrm{H}$ and 13C nuclear magnetic resonance study of carnosine, Canadian Journal of Chemistry 64, 2132-2138.

16. Branham, M. L., Singh, P., Bisetty, K., Sabela, M., and Govender, T. (2011) Preparation, spectrochemical, and computational analysis of L-carnosine (2-[(3aminopropanoyl)amino]-3-(1H-imidazol-5-yl)propanoic acid) and its ruthenium (II) coordination complexes in aqueous solution, Molecules 16, 10269-10291.

17. Gaggelli, E., and Valensin, G. (1990) 1H and 13C NMR relaxation investigation of the calcium complex of $\beta$-alanyl-L-histidine (carnosine) in aqueous solution, Journal of the Chemical Society, Perkin Transaction 2, 401-406.

18. Govindaraju, V., Young, K., and Maudsley, A. A. (2000) Proton NMR chemical shifts and coupling constants for brain metabolites, NMR in Biomedicine 13, 129-153.

19. Chevrier, B., Schalk, C., D'Orchymont, H., Rondeau, J.-M., Moras, D., and Tarnus, C. (1994) Crystal structure of Aeromonas proteolytica aminopeptidase: A prototypical member of the co-catalytic zinc enzyme family, Structure 2, 283-291.

20. Gilboa, R., Greenblatt, H. M., Perach, M., Spungin-Bialik, A., Lessel, U., Wohlfahrt, G., Schomburg, D., Blumberg, S., and Shoham, G. (2000) Interactions of Streptomyces griseus aminopeptidase with a methionine product analogue: A structural study at $1.53 \AA$ resolution, Acta Crystallographica Section D: Biological Crystallography 56, 551-558.

21. Jozic, D., Bourenkow, G., Bartunik, H., Scholze, H., Dive, V., Henrich, B., Huber, R., Bode, W., and Maskos, K. (2002) Crystal structure of the dinuclear zinc aminopeptidase PepV from Lactobacillus delbrueckii unravels its preference for dipeptides, Structure 10, 1097-1106.

22. Rowsell, S., Pauptit, R. A., Tucker, A. D., Melton, R. G., Blow, D. M., and Brick, P. (1997) Crystal structure of carboxypeptidase G2, a bacterial enzyme with applications in cancer therapy, Structure 5, 337-347.

23. Lundgren, S., Andersen, B., Piškur, J., and Dobritzsch, D. (2007) Crystal structures of yeast $\beta$-alanine synthase complexes reveal the mode of substrate binding and large scale domain closure movements, Journal of Biological Chemistry 282, 36037-36047.

24. Vistoli, G., Pedretti, A., Cattaneo, M., Aldini, G., and Testa, B. (2006) Homology modeling of human serum carnosinase, a potential medicinal target, and md simulations of its allosteric activation by citrate, Journal of Medicinal Chemistry 49, 3269-3277. 